\title{
Beyond cash-additive risk measures: when changing the numéraire fails
}

\section{Journal Article}

\section{Author(s):}

Farkas, Walter; Koch-Medina, Pablo; Munari, Cosimo

Publication date:

2014-01

Permanent link:

https://doi.org/10.3929/ethz-b-000075102

\section{Rights / license:}

In Copyright - Non-Commercial Use Permitted

\section{Originally published in:}

Finance and Stochastics 18(1), https://doi.org/10.1007/s00780-013-0220-9 


\title{
Beyond cash-additive risk measures: when changing the numéraire fails
}

\author{
Walter Farkas • Pablo Koch-Medina • \\ Cosimo Munari
}

Received: 31 May 2012 / Accepted: 5 September 2013 / Published online: 29 November 2013

(C) Springer-Verlag Berlin Heidelberg 2013

\begin{abstract}
We discuss risk measures representing the minimum amount of capital a financial institution needs to raise and invest in a pre-specified eligible asset to ensure it is adequately capitalized. Most of the literature has focused on cash-additive risk measures, for which the eligible asset is a risk-free bond, on the grounds that the general case can be reduced to the cash-additive case by a change of numéraire. However, discounting does not work in all financially relevant situations, especially when the eligible asset is a defaultable bond. In this paper, we fill this gap by allowing general eligible assets. We provide a variety of finiteness and continuity results for the corresponding risk measures and apply them to risk measures based on value-atrisk and tail value-at-risk on $L^{p}$ spaces, as well as to shortfall risk measures on Orlicz spaces. We pay special attention to the property of cash subadditivity, which has been recently proposed as an alternative to cash additivity to deal with defaultable bonds. For important examples, we provide characterizations of cash subadditivity and show that when the eligible asset is a defaultable bond, cash subadditivity is the exception rather than the rule. Finally, we consider the situation where the eligible asset is not liquidly traded and the pricing rule is no longer linear. We establish when the resulting risk measures are quasiconvex and show that cash subadditivity is only compatible with continuous pricing rules.
\end{abstract}

W. Farkas · P. Koch-Medina

Department of Banking and Finance, University of Zurich, Zurich, Switzerland

W. Farkas

e-mail: walter.farkas@bf.uzh.ch

P. Koch-Medina

e-mail: pablo.koch@bf.uzh.ch

W. Farkas $\cdot$ C. Munari $(\varangle)$

Department of Mathematics, ETH Zurich, Zurich, Switzerland

e-mail: cosimo.munari@math.ethz.ch 
Keywords Risk measures · Acceptance sets · General eligible assets · Defaultable bonds · Cash subadditivity · Quasiconvexity · Value-at-risk · Tail value-at-risk · Shortfall risk

Mathematics Subject Classification (2010) 91B30 - 46B42 • 46B40 • 46A55 • 06F30

JEL Classification $\mathrm{C} 60 \cdot \mathrm{G} 11 \cdot \mathrm{G} 22$

\section{Introduction}

Motivation Risk measures in their current axiomatic form were essentially introduced by Artzner, Delbaen, Eber and Heath in their landmark paper [4]. In that paper, the authors consider a one-period economy with dates $t=0$ and $t=T$ where future financial positions, or net worths, are represented by elements of the space $\mathscr{X}$ of random variables on a finite measurable space. A financial institution with future net worth $X \in \mathscr{X}$ is considered to be adequately capitalized if $X$ belongs to a prespecified set $\mathscr{A} \subset \mathscr{X}$ satisfying the axioms of a (coherent) acceptance set. Once a reference asset $S=\left(S_{0}, S_{T}\right)$ with initial price $S_{0}>0$ and positive terminal payoff $S_{T} \in \mathscr{X}$ has been specified, the corresponding risk measure is defined by setting

$$
\rho_{\mathscr{A}, S}(X):=\inf \left\{m \in \mathbb{R}: X+\frac{m}{S_{0}} S_{T} \in \mathscr{A}\right\} .
$$

As articulated in [4], the idea behind risk measures is that "sets of acceptable future net worths are the primitive objects to be considered in order to describe acceptance or rejection of a risk. [...] given some 'reference instrument', there is a natural way to define a measure of risk by describing how close or how far from acceptance a position is".

In terms of capital adequacy, the interpretation is that, whenever finite and positive, the number $\rho_{\mathscr{A}, S}(X)$ represents the minimum amount of capital the institution needs to raise and invest in the reference asset to become adequately capitalized. If finite and negative, then $-\rho_{\mathscr{A}, S}(X)$ represents the maximum amount of capital the institution can return without compromising its capital adequacy.

The theory of coherent risk measures was extended to general probability spaces in Delbaen [12]. In that paper, the focus is on cash-additive risk measures, i.e., risk measures where the reference asset is the risk-free asset $S=\left(1,1_{\Omega}\right)$ with risk-free rate set to zero. Hence, the risk measure is given by

$$
\rho_{\mathscr{A}}(X):=\rho_{\mathscr{A}, S}(X)=\inf \left\{m \in \mathbb{R}: X+m 1_{\Omega} \in \mathscr{A}\right\} .
$$

In the remark after Definition 2.1, Delbaen refers to [4] for an interpretation and notes that "here we are working in a model without interest rate, the general case can 'easily' be reduced to this case by 'discounting'".

The theory of risk measurement has since then been extended in many directions and, not surprisingly, based on the above discounting argument, most of the literature 
has focused on cash-additive risk measures. Yet, this exclusive focus on cash additivity is only justified if every economically meaningful situation can be reduced to the cash-additive setting. This, however, is by no means the case.

To see this, it is useful to make the discounting argument explicit. Consider an infinite probability space $(\Omega, \mathcal{F}, \mathbb{P})$ and assume the space of future financial positions is $\mathscr{X}=L^{p}$ for some $0 \leq p \leq \infty$. Take an acceptance set $\mathscr{A} \subset L^{p}$ and a reference asset $S=\left(S_{0}, S_{T}\right)$, where $S_{T} \in L^{p}$ is a nonzero, positive terminal payoff. If $S_{T}$ is (essentially) bounded away from zero, i.e., $S_{T} \geq \varepsilon$ almost surely for some $\varepsilon>0$, we can use $S$ as the new numéraire and consider "discounted" positions $\widetilde{X}:=X / S_{T}$. Note that in this case, discounted positions still belong to $\mathscr{X}$. Setting $\tilde{\mathscr{A}}:=\left\{X / S_{T}: X \in \mathscr{A}\right\}$, it is easy to see that

$$
\rho_{\mathscr{A}, S}(X)=S_{0} \rho_{\mathscr{A}}(\tilde{X}) .
$$

Hence, in this case the risk measure $\rho_{\mathscr{A}, S}$ can be reduced to a cash-additive risk measure acting on "discounted" positions. However, this reduction fails whenever the payoff of the reference asset is not bounded away from zero:

1. If $\mathbb{P}\left(S_{T}=0\right)>0$, then $S$ does not qualify as a numéraire and the "discounting" procedure is not applicable.

2. If $\mathbb{P}\left(S_{T}=0\right)=0$, but $S_{T}$ is not bounded away from zero, then we can use $S$ as a numéraire, but "discounted" positions will typically no longer belong to $L^{p}$, unless $p=0$. Moreover, any choice of the space of discounted positions will depend on the particular choice of the numéraire asset.

Reference assets whose payoffs are not bounded away from zero arise in situations which are not uncommon in financial applications. For instance, the payoff of shares is typically modeled by random variables which are not bounded away from zero, such as random variables with lognormal or exponential Lévy distribution. Perhaps more importantly, the same is true of defaultable bonds. Indeed, assume $S=\left(S_{0}, S_{T}\right)$ is a defaultable bond with face value 1 and price $S_{0}<1$. The payoff $S_{T}$ corresponds to a random variable taking values in the interval $[0,1]$ and can be interpreted as the recovery rate. Depending on what the recovery rate is in the various states of the economy, $S_{T}$ can be bounded away from zero or not, and can even assume the value zero in some future scenario. In particular, the case of zero recovery might describe the situation when actual recovery is positive, but occurs only after time $t=T$.

Bearing in mind the above mentioned interpretation given in [4], it is clear that acceptability is the key concept and that when measuring the distance to acceptability, we should not restrict a priori the range of possible reference assets. Therefore, it is important to go beyond cash-additive risk measures and to investigate risk measures with respect to a general reference asset whose payoff is not necessarily bounded away from zero. Moreover, we consider acceptance sets that are not necessarily coherent or convex. This allows us to cover, for example, risk measures based on valueat-risk acceptability, which is the most widely used acceptability criterion in practice.

Setting and main results In this paper, the space $\mathscr{X}$ of financial positions at time $t=T$ is assumed to be a general ordered topological vector space with posi- 
tive cone $\mathscr{X}_{+}$. The set $\mathscr{A}$ of acceptable future positions is taken to be any nontrivial subset of $\mathscr{X}$ satisfying $\mathscr{A}+\mathscr{X}_{+} \subset \mathscr{A}$, and the reference asset $S=\left(S_{0}, S_{T}\right)$ is described by its unit price $S_{0}>0$ and its nonzero terminal payoff $S_{T} \in \mathscr{X}_{+}$. This setup is general enough to cover the whole range of spaces commonly encountered in the literature, and to incorporate all financially relevant situations that cannot be captured within the standard cash-additive framework.

A comment on our choice to work on general topological vector spaces is in order. Since the typical spaces used in applications $-L^{p}$ and Orlicz spaces-are Fréchet lattices, one might argue that it is sufficient to restrict attention to this type of spaces. The motivation for a more abstract setting is twofold. First, there is a genuine mathematical interest in understanding the minimal structure required to support a theory of risk measures. Second, even when working within a Fréchet lattice setting, one is sometimes led to equip the underlying space with a different topology-for instance, to obtain the special dual representations in Biagini and Frittelli [7] or in Orihuela and Ruiz Galan [28] or, in particular, to deal with risk measures on $L^{\infty}$ having the Fatou property, which is simply lower semicontinuity with respect to the $\sigma\left(L^{\infty}, L^{1}\right)$ topology. This immediately takes us outside the domain of Fréchet lattices.

In this general context, we address the following issues:

Finiteness. Given our interpretation of risk measures as required capital, it is important to study finiteness properties. Indeed, if $\rho_{\mathscr{A}, S}(X)=\infty$ for a position $X \in \mathscr{X}$, then $X$ cannot be made acceptable by raising any amount of capital and investing it in the reference asset $S$. This means that $S$ is not an effective vehicle to help reach acceptability for that position. On the other hand, if $\rho_{\mathscr{A}, S}(X)=-\infty$, then we could extract arbitrary amounts of capital without compromising the acceptability of $X$, which is financially implausible. Note also that in many cases it is possible to establish that finiteness implies continuity - as for convex risk measures on Fréchet lattices due to the extended Namioka-Klee theorem in Biagini and Frittelli [7] or, in a more general setting, in Borwein [8]. Thus, understanding finiteness is also relevant from this perspective. Note that since no finiteness result is provided in [7], our finiteness results can be considered to be complementary to that paper.

Continuity and dual representations. Much effort in the literature has been devoted to showing various continuity properties of risk measures. From a practical perspective, continuity is important since if $\rho_{\mathscr{A}, S}$ fails to be continuous at some position $X$, then a slight change or misstatement of $X$ might lead to a dramatically different capital requirement. Moreover, as recently discussed in Krätschmer et al. [27], continuity is closely related to statistical robustness. Finally, continuity is also a useful property in the context of dual representations, which play an important role in optimization problems, for instance arising in connection to portfolio selection.

We undertake a systematic investigation of finiteness and continuity in terms of the interplay between the two fundamental financial primitives: the acceptance set $\mathscr{A}$ and the reference asset $S=\left(S_{0}, S_{T}\right)$. Since we do not restrict their range a priori, the results in this paper are entirely new in this generality and sometimes provide new insights even for the standard cash-additive case. The main results are the following:

1. In Proposition 3.1, we provide a complete picture of finiteness and continuity when $S_{T}$ belongs to the core or the interior of $\mathscr{X}_{+}$, without any assumption on $\mathscr{A}$. 
2. In Theorem 3.5, we establish a sufficient condition for finiteness in case $\mathscr{X}$ is a topological Riesz space and $\mathscr{A}$ has nonempty interior, extending to the nonconvex case the finiteness result obtained in Theorem 2.3 in Svindland [31] and in Theorem 4.6 in Cheridito and $\mathrm{Li}$ [11] for convex, cash-additive risk measures on $L^{p}$ spaces and Orlicz hearts, respectively.

3. In Theorem 3.10, we prove a characterization of continuity for convex risk measures, which can be seen as a generalization to arbitrary ordered topological vector spaces of the extended Namioka-Klee theorem in Biagini and Frittelli [7] when applied to risk measures.

4. In Theorem 3.12 and Corollary 3.14, we provide criteria for finiteness and continuity in case $\mathscr{A}$ is convex.

5. In Theorem 3.16, we provide a full characterization of finiteness and continuity when $\mathscr{A}$ is coherent.

Applications. Throughout Sect. 4 we provide several concrete examples. In particular, we focus on risk measures based on the most prominent acceptability criteria in practice: acceptability based on value-at-risk, on tail value-at-risk, and on shortfall risk arising in the context of utility maximization problems.

Cash subadditivity. Cash-subadditive risk measures were introduced in El Karoui and Ravanelli [16] with the intent to "model stochastic and/or ambiguous interest rates or defaultable contingent claims". Since our framework provides a natural approach to deal with defaultable reference assets, we investigate in Sect. 5.1 when $\rho_{\mathscr{A}, S}$ is cash subadditive on $L^{p}$. When $S=\left(S_{0}, S_{T}\right)$ is a defaultable bond, we always have cash subadditivity if $S$ can only default on the interest payment, i.e., if $\mathbb{P}\left(S_{T}<S_{0}\right)=0$. For important choices of the acceptance set, we show that $\rho_{\mathscr{A}, S}$ fails to be cash subadditive unless the probability $\mathbb{P}\left(S_{T}<S_{0}\right)$ that the invested capital is at risk is sufficiently small or sometimes even zero. Hence, if $\rho_{\mathscr{A}, S}$ is to be cash subadditive, the bond $S$ can only be allowed to default to a fairly limited extent. These findings provide a better insight into the property of cash subadditivity and show that the link between cash subadditivity and defaultability is less straightforward than suggested in [16].

Illiquid markets. In Sect. 5.2, we allow the possibility that the market for the reference asset is not liquid. In this case, we are naturally led to a quasiconvex risk measure, for which we provide in Proposition 5.11 a dual representation highlighting the underlying financial fundamentals. We also show in Proposition 5.13 that the associated risk measure can only be cash subadditive if the pricing rule for the reference asset depends continuously on the traded volume.

Embedding in the literature. Risk measures with respect to a general reference asset have been considered before to various degrees. In addition to the seminal paper [4] by Artzner et al., we refer to Jaschke and Küchler [24], Frittelli and Scandolo [21], Hamel [22], and Filipović and Kupper [18]. More recent relevant publications are the papers [5] by Artzner, Delbaen and Koch-Medina, and [26] by Konstantinides and Kountzakis. Some of these references contain results on finiteness and continuity, as well as dual representations. However, all relevant results are obtained, implicitly or explicitly, under the assumption that the payoff of the reference asset is an interior point of the positive cone. This critically limits their applicabil- 
ity since the positive cone of many spaces encountered in the literature has empty interior-for instance $L^{p}$ spaces, $0 \leq p<\infty$, and Orlicz hearts on nonatomic probability spaces. In this respect, Proposition 3.1 can be seen as a general formulation of that type of results. In [17], the present authors consider general eligible assets in the $L^{\infty}$ setting. However, the treatment there relies heavily on the fact that the positive cone in $L^{\infty}$ has nonempty interior, and it cannot be adapted to more general spaces which are important in financial applications. Finally, we mention that risk measures of the form $\rho_{\mathscr{A}}, S$ on $L^{p}$ can be regarded as scalarizations of set-valued risk measures — as studied in Hamel et al. [23]—where the underlying market consists of $S$ and the risk-free asset. Hence, our results can also be applied in that particular setting.

\section{Risk measures beyond cash additivity}

We start by defining risk measures associated to general acceptance sets and general reference assets, setting the scene for the remainder of the paper.

\subsection{The space of financial positions}

In this paper, financial positions are assumed to belong to a (Hausdorff) topological vector space over $\mathbb{R}$ denoted by $\mathscr{X}$. We assume $\mathscr{X}$ is ordered by a pointed convex cone $\mathscr{X}_{+}$called the positive cone. Note that a set $\mathscr{A} \subset \mathscr{X}$ is a cone if $\lambda \mathscr{A} \subset \mathscr{A}$ for all $\lambda \geq 0$ and is pointed if $\mathscr{A} \cap(-\mathscr{A})=\{0\}$. We write $X \leq Y$ whenever $Y-X \in \mathscr{X}_{+}$. The topological dual of $\mathscr{X}$ is denoted by $\mathscr{X}^{\prime}$. The space $\mathscr{X}^{\prime}$ is itself an ordered vector space when equipped with the positive cone $\mathscr{X}_{+}^{\prime}$ consisting of all functionals $\psi \in \mathscr{X}^{\prime}$ such that $\psi(X) \geq 0$ whenever $X \in \mathscr{X}_{+}$.

If $\mathscr{A}$ is a subset of $\mathscr{X}$, we denote by $\operatorname{int}(\mathscr{A}), \overline{\mathscr{A}}$ and $\partial \mathscr{A}$ the interior, the closure and the boundary of $\mathscr{A}$, respectively. Moreover, we denote by core $(\mathscr{A})$ the core, or algebraic interior, of $\mathscr{A}$, i.e., the set of all positions $X \in \mathscr{A}$ such that for each $Y \in \mathscr{X}$, there exists $\varepsilon>0$ with $X+\lambda Y \in \mathscr{A}$ whenever $|\lambda|<\varepsilon$.

In case $\mathscr{X}$ is equipped with a lattice structure, we use the standard notation $X \vee Y:=\sup \{X, Y\}$ and $X \wedge Y:=\inf \{X, Y\}$. Moreover, we set $X^{+}:=X \vee 0$ for the positive part of $X, X^{-}:=(-X) \vee 0$ for its negative part, and $|X|:=X \vee(-X)$ for its absolute value.

Example 2.1 (Standard spaces) Standard examples of ordered topological vector spaces used in financial mathematics are provided by spaces of random variables defined on a probability space $(\Omega, \mathcal{F}, \mathbb{P})$, which throughout this paper will always be assumed to be nonatomic. As usual, random variables which coincide almost surely are identified so that equalities and inequalities involving random variables will always be understood in the almost sure sense. The natural order structure is given by almost sure pointwise ordering. The vector space $L^{0}$ of all $\mathcal{F}$-measurable functions $X: \Omega \rightarrow \mathbb{R}$ is a Fréchet lattice with respect to the topology of convergence in probability. If $0<p<\infty$, we denote by $L^{p}$ the subspace of $L^{0}$ consisting of all functions 
satisfying $\mathbb{E}\left[|X|^{p}\right]<\infty$. It is a Banach lattice under the usual norm when $p \geq 1$, and a Fréchet lattice under the usual metric when $0<p<1$. The space $L^{\infty}$ is the subspace of $L^{0}$ consisting of all essentially bounded functions. It is a Banach lattice with respect to the standard (essential) supremum norm. If $\Phi$ is an Orlicz function as defined in [14], the Orlicz space $L^{\Phi}$ is the subspace of $L^{0}$ consisting of all functions $X \in L^{0}$ such that $\mathbb{E}[\Phi(\lambda X)]<\infty$ for some $\lambda>0$. The Orlicz heart $H^{\Phi}$ is the subspace of $L^{\Phi}$ consisting of all functions satisfying the previous inequality for every $\lambda>0$. These spaces are Banach lattices under the Luxemburg norm.

If $\mathscr{X}$ is any of the spaces described above and $\mathscr{Y}$ is a vector space such that $(\mathscr{X}, \mathscr{Y})$ is a dual pair, then $\mathscr{X}$ equipped with the weak topology $\sigma(\mathscr{X}, \mathscr{Y})$ is an ordered topological vector space. However, it is no longer a Fréchet lattice by Corollary 9.9 in [1]. A typical instance of this situation encountered in financial mathematics is when $L^{\infty}$ is equipped with the weak* topology $\sigma\left(L^{\infty}, L^{1}\right)$.

The positive cone $\mathscr{X}_{+}$may have empty interior. In this case, we consider two types of substitutes for interior points: order units and strictly positive elements. The elements in core $\left(\mathscr{X}_{+}\right)$are called order units. A point $X \in \mathscr{X}_{+}$is called strictly positive whenever $\psi(X)>0$ for all nonzero $\psi \in \mathscr{X}_{+}^{\prime}$. The set of all strictly positive points is denoted by $\mathscr{X}_{++}$. We always have $\operatorname{int}\left(\mathscr{X}_{+}\right) \subset \operatorname{core}\left(\mathscr{X}_{+}\right) \subset \mathscr{X}_{++}$. These inclusions are in general strict, but they coincide whenever $\mathscr{X}_{+}$has nonempty interior.

Example 2.2 (i) (Nonempty interior) The positive cone of $L^{\infty}$ has nonempty interior, and $X \in \operatorname{int}\left(L_{+}^{\infty}\right)$ if and only if $X \geq \varepsilon$ almost surely for some $\varepsilon>0$. In particular, one should not confuse strictly positive elements with functions that are strictly positive almost surely.

(ii) (Empty interior, nonempty core) If we endow $L^{\infty}$ with the weak ${ }^{*}$ topology $\sigma\left(L^{\infty}, L^{1}\right)$, then it is not difficult to see that the interior of the positive cone is empty. Note that as in (i), any positive element in $L^{\infty}$ which is bounded away from zero is an order unit. Moreover, the strictly positive elements are precisely those $X \in L^{\infty}$ such that $X>0$ almost surely. As a result, the inclusion core $\left(L_{+}^{\infty}\right) \subset L_{++}^{\infty}$ is strict, even if the positive cone has nonempty core.

(iii) (Empty core, but strictly positive elements) The positive cone of $L^{p}$, for $1 \leq p<\infty$, has empty core. However, the elements $X \in L^{p}$ such that $X>0$ almost surely correspond to the strictly positive elements. The same is true for any (nontrivial) Orlicz heart $H^{\Phi}$.

(iv) (No strictly positive elements) It is known that strictly positive elements may fail to exist; see Exercise 10 in Sect. 2.2 of [2], where the space $\mathscr{X}$ is a nonstandard function space. In Remark 4.8 below, we provide a more interesting example: We show that the Orlicz space $L^{\Phi}$ defined by $\Phi(x):=e^{|x|}-1$ has no strictly positive elements.

\subsection{From unacceptable to acceptable}

In this section, we introduce risk measures with respect to general reference assets and general acceptance sets and establish some of their basic properties. A detailed motivation for studying this type of risk measures was provided in the introduction. 
In Sect. 4, we discuss several examples of acceptance sets which are relevant for financial applications.

Definition 2.3 A set $\mathscr{A} \subset \mathscr{X}$ is called an acceptance set whenever the following two conditions are satisfied:

(i) $\mathscr{A}$ is a nonempty, proper subset of $\mathscr{X}$ (non-triviality);

(ii) if $X \in \mathscr{A}$ and $Y \geq X$, then $Y \in \mathscr{A}$ (monotonicity).

These conditions seem to be minimal in the sense that non-triviality allows to discriminate between "good" and "bad" positions, and monotonicity captures the intuition that a financial institution is better capitalized than another if the net worth of the first dominates the net worth of the second. Special classes of acceptance sets considered later are convex acceptance sets, conic acceptance sets, and coherent acceptance sets, i.e., acceptance sets which are convex cones. We refer to [4] and [19] for a financial interpretation of these special acceptance sets.

We assume the existence of a financial market where assets are liquidly traded. A traded asset will be represented by a pair $S=\left(S_{0}, S_{T}\right)$, where $S_{0}>0$ is the initial price of the asset and $S_{T} \in \mathscr{X}_{+}$its terminal payoff, which is always assumed to be nonzero. If a position $X \in \mathscr{X}$ is not acceptable with respect to a given acceptance set $\mathscr{A} \subset \mathscr{X}$, it is natural to ask which actions can turn it into an acceptable position, and at which cost. In line with the definition of a risk measure proposed in [4], we allow one specific action: Raising capital and investing it in a pre-specified traded asset $S$. In the sequel, we adopt the standard notation $\overline{\mathbb{R}}:=\mathbb{R} \cup\{\infty,-\infty\}$.

Definition 2.4 Let $\mathscr{A} \subset \mathscr{X}$ be a monotone set and $S=\left(S_{0}, S_{T}\right)$ a traded asset. The risk measure with respect to $\mathscr{A}$ and $S$ is the function $\rho_{\mathscr{A}, S}: \mathscr{X} \rightarrow \overline{\mathbb{R}}$ defined by

$$
\rho_{\mathscr{A}, S}(X):=\inf \left\{m \in \mathbb{R}: X+\frac{m}{S_{0}} S_{T} \in \mathscr{A}\right\} .
$$

The asset $S$ is called the eligible, or reference, asset.

When finite and positive, $\rho_{\mathscr{A}, S}(X)$ represents the "minimum" amount of capital that needs to be invested in the eligible asset and added to the position $X$ to reach acceptability. When negative, it represents the amount of capital that can be extracted from $X$ without compromising its acceptability. Clearly, unless $\mathscr{A}$ is closed, the infimum in (2.1) is not necessarily attained.

Before stating some natural properties of risk measures $\rho_{\mathscr{A}}, S$, we recall some notation and terminology for a map $\rho: \mathscr{X} \rightarrow \overline{\mathbb{R}}$. The (effective) domain of $\rho$ is the set

$$
\operatorname{dom}(\rho):=\{X \in \mathscr{X}: \rho(X)<\infty\} .
$$

If the epigraph epi $(\rho):=\{(X, \alpha) \in \mathscr{X} \times \mathbb{R}: \rho(X) \leq \alpha\}$ is convex, respectively conic, then $\rho$ is called convex, respectively positively homogeneous. The function $\rho$ is decreasing if $\rho(X) \geq \rho(Y)$ for all $X \leq Y$. Moreover, we say that $\rho$ is lower semicontinuous at $X \in \mathscr{X}$ if for every $\varepsilon>0$, there exists a neighborhood $\mathscr{U}$ of $X$ such that 
$\rho(Y) \geq \rho(X)-\varepsilon$ for all $Y \in \mathscr{U}$, and upper semicontinuous at $X$ when $-\rho$ is lower semicontinuous at $X$. Note that continuity is equivalent to having both lower and upper semicontinuity. If $S=\left(S_{0}, S_{T}\right)$ is a traded asset, the function $\rho$ is said to be $S$-additive if for any $X \in \mathscr{X}$,

$$
\rho\left(X+\lambda S_{T}\right)=\rho(X)-\lambda S_{0} \quad \text { for all } \lambda \in \mathbb{R} .
$$

The following lemma collects some basic facts about risk measures of the form $\rho_{\mathscr{A}, S}$, for which we also refer to [17].

Lemma 2.5 Let $\mathscr{A} \subset \mathscr{X}$ be a monotone set and $S=\left(S_{0}, S_{T}\right)$ a traded asset. Then $\rho_{\mathscr{A}, S}$ satisfies the following properties:

(i) $\rho_{\mathscr{A}, S}$ is $S$-additive and decreasing.

(ii) $\left\{X \in \mathscr{X}: \rho_{\mathscr{A}, S}(X)=0\right\} \subset \partial \mathscr{A}$ and

$$
\operatorname{int}(\mathscr{A}) \subset\left\{X \in \mathscr{X}: \rho_{\mathscr{A}, S}(X)<0\right\} \subset \mathscr{A} \subset\left\{X \in \mathscr{X}: \rho_{\mathscr{A}, S}(X) \leq 0\right\} \subset \overline{\mathscr{A}} .
$$

(iii) $\rho_{\mathscr{A}, S}$ is lower semicontinuous at $X$ if and only if $X+\frac{m}{S_{0}} S_{T} \notin \overline{\mathscr{A}}$ for any $m<\rho_{\mathscr{A}, S}(X)$.

(iv) $\rho_{\mathscr{A}, S}$ is upper semicontinuous at $X$ if and only if $X+\frac{m}{S_{0}} S_{T} \in \operatorname{int}(\mathscr{A})$ for any $m>\rho_{\mathscr{A}, S}(X)$.

(v) If $\mathscr{A}$ is convex, respectively conic, then $\rho_{\mathscr{A}, S}$ is convex, respectively positively homogeneous.

Remark 2.6 (i) By part (iv) in Lemma 2.5, the first inclusion in (2.2) is an equality if and only if $\rho_{\mathscr{A}, S}$ is globally upper semicontinuous. By part (iii), the last inclusion in (2.2) is an equality if and only if $\rho_{\mathscr{A}, S}$ is globally lower semicontinuous.

(ii) Note that $\rho_{\mathscr{A}, S}$ cannot be (upper semi) continuous at any point $X$ of its domain if $\operatorname{int}(\mathscr{A})$ is empty. This follows from part (iv) of Lemma 2.5.

(iii) Consider the space $L^{p}$ for some $1 \leq p<\infty$. By the previous point, Theorem 2.9 in [25] cannot be true in the stated generality, namely that any lower semicontinuous, coherent cash additive risk measure $\rho: L^{p} \rightarrow \mathbb{R} \cup\{\infty\}$ must automatically be finite-valued and continuous. To see this, consider the closed, coherent acceptance set $L_{+}^{p}$ and the risk-free asset $S=\left(1,1_{\Omega}\right)$. The corresponding risk measure $\rho_{L_{+}, S}$ is cash additive, convex and lower semicontinuous, but $\rho_{L_{+}, S}(X)=\infty$ whenever $X$ is not essentially bounded from below. Moreover, $\rho_{L_{+}^{p}, S}$ cannot be continuous at any point of finiteness since $L_{+}^{p}$ has empty interior. The problem in [25] originates with the proof of Proposition 2.8 in that paper which only works for finite-valued functions.

\section{The interplay between acceptance set and eligible asset}

In this section, we investigate finiteness and continuity properties of risk measures on general ordered topological vector spaces, highlighting the interplay between the acceptance set and the eligible asset. Essentially, the more we require from the acceptance set, the less we need to require from the eligible asset. 


\subsection{General acceptance sets}

Assume that $\mathscr{A} \subset L^{\infty}$ is an arbitrary acceptance set, and that the payoff $S_{T}$ of a traded asset $S=\left(S_{0}, S_{T}\right)$ is an interior point of $L_{+}^{\infty}$, i.e., $S_{T}$ is bounded away from zero. In this case, a standard argument shows that the corresponding risk measure $\rho_{\mathscr{A}, S}$ is finite-valued and continuous; see also [17]. For a general ordered topological vector space $\mathscr{X}$, the statement remains true. When the interior of the positive cone is empty, we can still obtain finiteness if we require that $S_{T}$ is an order unit.

Proposition 3.1 Let $\mathscr{A} \subset \mathscr{X}$ be an arbitrary acceptance set and $S=\left(S_{0}, S_{T}\right)$ a traded asset.

(i) If $S_{T} \in \operatorname{core}\left(\mathscr{X}_{+}\right)$, then $\rho_{\mathscr{A}, S}$ is finite-valued.

(ii) If $S_{T} \in \operatorname{int}\left(\mathscr{X}_{+}\right)$, then $\rho_{\mathscr{A}, S}$ is finite-valued and continuous.

Moreover, if $\mathscr{X}$ is an ordered normed space and $S_{T} \in \operatorname{int}\left(\mathscr{X}_{+}\right)$, then $\rho_{\mathscr{A}, S}$ is Lipschitz-continuous.

Proof (i) Fix $X \in \mathscr{X}$ and take $Y \in \mathscr{A}$ and $Z \in \mathscr{A}^{c}$. Since $S_{T}$ belongs to the core of $\mathscr{X}_{+}$, there exists $\lambda_{1}>0$ such that $Y-X \leq \lambda_{1} S_{T}$. As a result, we have $X+\lambda_{1} S_{T} \in \mathscr{A}$, implying $\rho_{\mathscr{A}, S}(X)<\infty$. On the other hand, we can also find $\lambda_{2}>0$ so that $X-Z \leq \lambda_{2} S_{T}$. Thus, $X-\lambda_{2} S_{T} \notin \mathscr{A}$ by monotonicity, showing that $\rho_{\mathscr{A}, S}(X)>-\infty$.

(ii) Since $S_{T}$ is also an element of the core of $\mathscr{X}_{+}$, finiteness follows from (i). To prove continuity, take an arbitrary $X \in \mathscr{X}$ and assume it is the limit of a net $\left(X_{\alpha}\right)$. Since $\left\{Y \in \mathscr{X}: S_{T} \leq Y \leq S_{T}\right\}$ is a neighborhood of zero, for every $\varepsilon>0$ there exists $\alpha_{\varepsilon}$ such that $-\varepsilon S_{T} \leq X_{\alpha}-X \leq \varepsilon S_{T}$ whenever $\alpha \geq \alpha_{\varepsilon}$. But then $\left|\rho_{\mathscr{A}, S}\left(X_{\alpha}\right)-\rho_{\mathscr{A}, S}(X)\right| \leq \varepsilon S_{0}$ for $\alpha \geq \alpha_{\varepsilon}$, showing that $\rho_{\mathscr{A}, S}$ is continuous at $X$.

Finally, assume $\mathscr{X}$ is an ordered normed space and $S_{T} \in \operatorname{int}\left(\mathscr{X}_{+}\right)$so that $\rho_{\mathscr{A}, S}$ is finite-valued by part (i). Using Theorem 9.40 in [1], it is not difficult to prove that $S_{T} \in \operatorname{int}\left(\mathscr{X}_{+}\right)$is equivalent to the existence of a constant $\lambda>0$ such that $X \leq \lambda\|X\| S_{T}$ for every nonzero $X \in \mathscr{X}$. To prove Lipschitz-continuity, take now two positions $X$ and $Y$ in $\mathscr{X}$. Since $Y \leq X+\lambda\|X-Y\| S_{T}$, we obtain $\rho_{\mathscr{A}, S}(X)-\rho_{\mathscr{A}, S}(Y) \leq \lambda S_{0}\|X-Y\|$. Exchanging $X$ and $Y$, we conclude the proof.

Remark 3.2 The above proposition is easily seen to hold if $\mathscr{X}_{+}$is only assumed to induce a pre-ordering on $\mathscr{X}$, i.e., if $\mathscr{X}_{+}$is a convex cone which is not necessarily pointed. This will be important in Theorem 3.16 where the proposition is applied with respect to the pre-ordering induced by a coherent acceptance set.

We now turn to general acceptance sets in topological Riesz spaces, i.e., topological vector spaces equipped with a lattice ordering. First we need the following generalization of the notion of order units. An element $Z \in \mathscr{X}_{+}$in a Riesz space $\mathscr{X}$ is called a weak topological unit if for every $X \in \mathscr{X}_{+}$, we have $X \wedge n Z \rightarrow X$ as $n \rightarrow \infty$.

The next technical lemma extends Theorem 6.3 in [29] beyond the normed space setting and establishes the link between weak topological units and strictly positive 
elements. Recall that a topological Riesz space $\mathscr{X}$ is said to be locally solid when there exists a neighborhood base of zero consisting of solid neighborhoods $\mathscr{U}$, i.e., satisfying $X \in \mathscr{U}$ whenever $Y \in \mathscr{U}$ and $|X| \leq|Y|$. For more details, we refer to Chap. 9 in [1].

Lemma 3.3 Let $\mathscr{X}$ be a topological Riesz space. Then every weak topological unit is strictly positive. If $\mathscr{X}$ is also locally convex and locally solid, the converse is true as well.

Proof Let $Z$ be a weak topological unit and assume $\psi(Z)=0$ for some nonzero $\psi \in \mathscr{X}_{+}^{\prime}$. Then $\psi(X \wedge n Z)=0$ for all $X \in \mathscr{X}_{+}$and all positive integers $n$. Hence by continuity, $\psi(X)=0$ for all $X \in \mathscr{X}_{+}$, implying that $\psi$ is null. This proves $Z$ must be strictly positive.

Assume now that $\mathscr{X}$ is locally convex. Let $Z$ be strictly positive and take $X \in \mathscr{X}_{+}$. To prove that $Z$ is a weak topological unit, it is sufficient to show that for every solid neighborhood of zero $\mathscr{U}$ we eventually have $X-(X \wedge n Z) \in \mathscr{U}$. By Theorem 8.54 in [1], the principal ideal $\mathcal{I}_{Z}:=\{Y \in \mathscr{X}: \exists \lambda>0$ with $|Y| \leq \lambda Z\}$ is weakly dense in $\mathscr{X}$. Since $\mathcal{I}_{Z}$ is convex and $\mathscr{X}$ is locally convex, this implies that $\mathcal{I}_{Z}$ is dense in $\mathscr{X}$ with respect to the original topology. As a result, we can find $Y \in \mathcal{I}_{Z}$ with $X-Y \in \mathscr{U}$. Setting $W:=X \wedge Y^{+}$and noting that $W$ belongs to $\mathcal{I}_{Z}$, we see that $W \leq n_{0} Z$ for some positive integer $n_{0}$. Since for all $n \geq n_{0}$

$$
0 \leq X-(X \wedge n Z) \leq X-\left(X \wedge n_{0} Z\right) \leq X-W \leq X \vee Y-X \wedge Y=|X-Y| \text {, }
$$

the solidity of $\mathscr{U}$ implies that $X-(X \wedge n Z) \in \mathscr{U}$ for every $n \geq n_{0}$, concluding the proof.

Remark 3.4 (i) Weak topological units differ from weak order units $Z \in \mathscr{X}_{+}$which satisfy $X=\sup _{n}(X \wedge n Z)$ for all $X \in \mathscr{X}_{+}$. For instance, every element $Z \in L_{+}^{\infty}$ with $Z>0$ almost surely is a weak order unit, but not a weak topological unit unless it is bounded away from zero.

(ii) By the previous result, weak topological units in $L^{p}$ spaces, $1 \leq p<\infty$, or in Orlicz hearts are precisely those positive elements $Z$ for which $Z>0$ almost surely. In $L^{\infty}$, they correspond to elements that are bounded away from zero.

(iii) Recall that $L^{p}$ is a topological Riesz space which is not locally convex whenever $0 \leq p<1$. In this case, the set of strictly positive elements coincides with the positive cone since the only continuous linear functional is the zero functional. However, it is not difficult to show that $Z \in L_{+}^{p}$ is a weak topological unit if and only if $Z>0$ almost surely.

The next theorem is the main result of this section and provides a sufficient condition for a risk measure on a topological Riesz space to be finite-valued. We require neither convexity of $\mathscr{A}$ nor cash additivity of $\rho_{\mathscr{A}, S}$. Our result contains as a special case non-convex extensions of two well-known finiteness results for convex cashadditive risk measures: Theorem 2.3 in Svindland [31] on $L^{p}$ spaces, $1 \leq p \leq \infty$, and Theorem 4.6 in Cheridito and Li [11] on Orlicz hearts. The proofs of both of 
these results rely on separation arguments which cannot be reproduced in our nonconvex setting. In fact, our approach is simpler and depends solely on the lattice structure. It is closer in spirit to the proof of Proposition 6.7 in Shapiro et al. [30] who, however, make use of a category argument that only works if lower semicontinuity is additionally assumed.

Theorem 3.5 Let $\mathscr{X}$ be a topological Riesz space and $\mathscr{A} \subset \mathscr{X}$ an acceptance set with nonempty interior. Let $S=\left(S_{0}, S_{T}\right)$ be a traded asset and assume that $\rho_{\mathscr{A}, S}$ does not attain the value $-\infty$. If $S_{T}$ is a weak topological unit, then $\rho_{\mathscr{A}, S}$ is finite-valued.

Proof Take $Z \in \operatorname{int}(\mathscr{A})$ and choose a neighborhood of zero $\mathscr{U}$ with $Z+\mathscr{U} \subset \mathscr{A}$. Fix $Y \in \mathscr{X}_{+}$and note that $Y=\left(Y \wedge n S_{T}\right)+\left(Y-n S_{T}\right)^{+}$for any $n \in \mathbb{N}$. Since $S_{T}$ is a weak topological unit, $\left(Y-n S_{T}\right)^{+} \rightarrow 0$ as $n \rightarrow \infty$ so that $-\left(Y-m S_{T}\right)^{+} \in \mathscr{U}$ for sufficiently large $m$. Note that $Z-\left(Y-m S_{T}\right)^{+}-m S_{T} \leq Z-Y$ and $Z-\left(Y-m S_{T}\right)^{+} \in \mathscr{A}$. Hence monotonicity and $S$-additivity yield $\rho_{\mathscr{A}, S}(Z-Y) \leq$ $m S_{0}<\infty$. Now take an arbitrary $X \in \mathscr{X}$. Setting $Y:=(Z-X)^{+}$gives $\rho_{\mathscr{A}, S}(X) \leq$ $\rho_{\mathscr{A}, S}(Z-Y)<\infty$. Hence $\rho_{\mathscr{A}, S}$ is finite-valued.

When $\mathscr{X}$ is a Fréchet lattice, i.e., a topological Riesz space which is locally solid and completely metrizable, the interior and the core of a monotone set always coincide. This can be shown by adapting the proof of Lemma 4.1 for monotone functionals on a Banach lattice in Cheridito and Li [11]. Consequently, on Fréchet lattices the above theorem holds under the weaker assumption that the acceptance set has nonempty core. Because of its practical relevance-it is generally easier to show that an element belongs to the core than to show it belongs to the interior of a set-we record this in the next proposition.

Proposition 3.6 Let $\mathscr{X}$ be a Fréchet lattice. The following statements hold:

(i) $\operatorname{int}(\mathscr{A})=\operatorname{core}(\mathscr{A})$ for every monotone set $\mathscr{A} \subset \mathscr{X}$.

(ii) If $\mathscr{A}$ is an acceptance set with nonempty core and $S=\left(S_{0}, S_{T}\right)$ a traded asset with $S_{T}$ weak topological unit, then $\rho_{\mathscr{A}, S}$ is finite-valued whenever it does not attain the value $-\infty$.

Remark 3.7 (i) Part (i) of the above proposition provides an alternative approach to the extended Namioka-Klee theorem obtained in Biagini and Frittelli [7]: Every convex monotone map $\rho: \mathscr{X} \rightarrow \mathbb{R} \cup\{\infty\}$ on a Fréchet lattice $\mathscr{X}$ is continuous on the interior of its domain. Indeed, assume $\rho$ is such a map and let $X$ be an interior point of its domain. As in the proof of Proposition 3.8 below, it is not difficult to show that for any $\alpha>\rho(X)$, the point $X$ belongs to the core of $\mathscr{A}:=\{Y \in \mathscr{X}: \rho(Y)<\alpha\}$. Then $X$ is an interior point of $\mathscr{A}$ by Proposition 3.6, hence the map $\rho$ turns out to be bounded from above on a neighborhood of $X$. As a result, Theorem 5.43 in [1] implies $\rho$ is continuous at $X$.

(ii) If the acceptance set in the preceding proposition is additionally assumed to be convex, the finiteness of $\rho_{\mathscr{A}, S}$ immediately implies continuity by Theorem 1 in [7] or by the first remark. 


\subsection{Convex acceptance sets}

In this section, we focus on convex acceptance sets and provide a variety of finiteness and continuity results in general ordered topological vector spaces. Convexity allows us to obtain results for a wide range of eligible assets, without requiring that the positive cone has nonempty interior. In particular, all results in this section apply to $L^{p}$, $1 \leq p \leq \infty$, and Orlicz spaces. We start by showing a general necessary condition for a convex risk measure to be finite.

Proposition 3.8 Let $\mathscr{A} \subset \mathscr{X}$ be a convex acceptance set and $S=\left(S_{0}, S_{T}\right)$ a traded asset. Assume that $\rho_{\mathscr{A}, S}$ does not attain the value $-\infty$. Then $\operatorname{core}\left(\operatorname{dom}\left(\rho_{\mathscr{A}}, S\right)\right)$ is nonempty if and only if core $(\mathscr{A})$ is nonempty. In particular, if $\rho_{\mathscr{A}, S}$ is finite-valued, then core $(\mathscr{A})$ is nonempty.

Proof Since $\mathscr{A} \subset \operatorname{dom}\left(\rho_{\mathscr{A}}, S\right)$, it is enough to prove the "only if" part. Suppose that $X \in \operatorname{core}\left(\operatorname{dom}\left(\rho_{\mathscr{A}}, S\right)\right)$ and assume without loss of generality that $\rho_{\mathscr{A}, S}(X)<0$. Take a nonzero $Y \in \mathscr{X}$ and choose $\varepsilon>0$ in such a way that $X+\lambda Y \in \operatorname{dom}\left(\rho_{\mathscr{A}, S}\right)$ whenever $\lambda \in(-\varepsilon, \varepsilon)$. Then $f(\lambda):=\rho_{\mathscr{A}, S}(X+\lambda Y)$ defines a real-valued function on $(-\varepsilon, \varepsilon)$, which must be continuous by convexity. Since $f(0)=\rho_{\mathscr{A}, S}(X)<0$, it follows that there exists $\delta>0$ such that $\rho_{\mathscr{A}, S}(X+\lambda Y)=f(\lambda)<0$ for $\lambda \in(-\delta, \delta)$, and consequently, $X+\lambda Y \in \mathscr{A}$ for all such $\lambda$. In conclusion, $X \in \operatorname{core}(\mathscr{A})$.

Remark 3.9 If $\mathscr{X}$ is a Fréchet lattice and $\mathscr{A} \subset \mathscr{X}$ a convex acceptance set, it follows immediately from the above result and Proposition 3.6 that the domain of a risk measure $\rho_{\mathscr{A}, S}$ has nonempty interior if and only if $\mathscr{A}$ itself has nonempty interior.

The preceding remark allows us to reformulate the continuity part of Theorem 1 in Biagini and Frittelli [7] when restricted to convex risk measures as follows: Let $\mathscr{X}$ be a Frechét lattice, $\mathscr{A} \subset \mathscr{X}$ a convex acceptance set with nonempty interior and $S=\left(S_{0}, S_{T}\right)$ a traded asset. If $\rho_{\mathscr{A}, S}$ does not assume the value $-\infty$, then it is continuous on the interior of its domain. As a consequence, the following result can be regarded as an extended Namioka-Klee theorem for convex risk measures defined on general ordered topological vector spaces. Note that no lattice structure is required here and the proof is more direct.

Theorem 3.10 Let $\mathscr{A} \subset \mathscr{X}$ be a convex acceptance set and $S=\left(S_{0}, S_{T}\right)$ a traded asset. Assume $\rho_{\mathscr{A}, S}$ does not take the value $-\infty$. The following statements are equivalent:

(a) $\operatorname{dom}\left(\rho_{\mathscr{A}, S}\right)$ has nonempty interior and $\rho_{\mathscr{A}, S}$ is continuous on $\operatorname{int}\left(\operatorname{dom}\left(\rho_{\mathscr{A}, S}\right)\right)$.

(b) $\operatorname{int}(\mathscr{A})$ is nonempty.

In particular, if $\mathscr{A}$ has nonempty interior, then $\rho_{\mathscr{A}, S}$ is continuous on $\mathscr{X}$ whenever it is finite-valued.

Proof By Remark 2.6, it is enough to prove that (b) implies (a). Note first that the domain of $\rho_{\mathscr{A}, S}$ has nonempty interior because it contains $\mathscr{A}$. Since $\rho_{\mathscr{A}, S}$ is bounded above by 0 on $\operatorname{int}(\mathscr{A})$, we can apply Theorem 5.43 in [1] to obtain (a). 
We now focus on finiteness results in the context of convex acceptance sets with nonempty interior. In this case, finiteness always implies continuity by Theorem 3.10. The following lemma will prove to be useful.

Lemma 3.11 Let $\mathscr{A} \subset \mathscr{X}$ be an arbitrary acceptance set and consider a (not necessarily continuous) linear functional $\psi: \mathscr{X} \rightarrow \mathbb{R}$. Then $\psi$ is positive whenever it is bounded from below on $\mathscr{A}$.

Proof Let $X \in \mathscr{X}_{+}$be arbitrary and fix $Y \in \mathscr{A}$. Then by monotonicity of $\mathscr{A}$, we have $Y+\lambda X \in \mathscr{A}$ for all $\lambda \geq 0$. Hence, $\psi(Y)+\lambda \psi(X) \geq \inf _{Z \in \mathscr{A}} \psi(Z)>-\infty$ for all $\lambda \geq 0$, which can only be true if $\psi(X) \geq 0$.

We start by showing that if a risk measure is finite-valued in the direction of some strictly positive element, then it is finite-valued on $\mathscr{X}$. This provides a simple criterion for finiteness and continuity which we use in Proposition 4.6 in the context of shortfall risk measures. Note that we do not require any explicit assumption on the eligible asset $S$.

Theorem 3.12 Assume $\mathscr{X}$ admits a strictly positive element $U \in \mathscr{X}_{+}$. Let $\mathscr{A} \subset \mathscr{X}$ be a convex acceptance set with nonempty interior and $S=\left(S_{0}, S_{T}\right)$ a traded asset. Assume $\rho_{\mathscr{A}, S}$ does not attain the value $-\infty$. Then $\rho_{\mathscr{A}, S}$ is finite-valued if and only if $\rho_{\mathscr{A}, S}(-\lambda U)<\infty$ for all $\lambda>0$. In this case, $\rho_{\mathscr{A}, S}$ is also continuous.

Proof We only need to prove the "if" part. Assume $X \notin \operatorname{dom}\left(\rho_{\mathscr{A}, S}\right)$. Since $\operatorname{dom}\left(\rho_{\mathscr{A}, S}\right)$ is convex and has nonempty interior, by separation and Lemma 3.11 we find a nonzero $\psi \in \mathscr{X}_{+}^{\prime}$ with $\psi(X) \leq \psi(-\lambda U)$ for all $\lambda>0$. But this implies $\psi(U)=0$, contradicting the strict positivity of $U$. Hence, $\rho_{\mathscr{A}, S}$ must be finite-valued and, hence, also continuous.

Remark 3.13 (i) Theorem 3.12 is particularly useful when $\mathscr{X}$ is an $L^{p}$ space with $1 \leq p \leq \infty$, or an Orlicz heart, since $U:=1_{\Omega}$ is a strictly positive element in these spaces.

(ii) Note that if the acceptance set in the preceding theorem is assumed to be coherent, the condition $\rho_{\mathscr{A}, S}(-\lambda U)<\infty$ for all $\lambda>0$ becomes equivalent to $\rho_{\mathscr{A}, S}(-U)<\infty$ due to positive homogeneity.

By Proposition 3.1, for general acceptance sets we always have finiteness if the payoff of the eligible asset is an order unit. If the acceptance set is convex and has nonempty interior, it suffices to require that the payoff of the eligible asset is strictly positive. In Proposition 4.6 below, we show that this condition is sometimes also necessary for finiteness. Note that in contrast to other results in this section, we do not need to require a priori that the risk measure does not attain the value $-\infty$.

Corollary 3.14 Let $\mathscr{A} \subset \mathscr{X}$ be a convex acceptance set with nonempty interior, and $S=\left(S_{0}, S_{T}\right)$ a traded asset. If $S_{T}$ is strictly positive, then $\rho_{\mathscr{A}, S}$ is finite-valued and continuous. 
Proof First, we show that $\rho_{\mathscr{A}, S}$ never attains the value $-\infty$. Indeed, assume to the contrary that $\rho_{\mathscr{A}, S}(X)=-\infty$ for some $X \in \mathscr{X}$, and take $Y \notin \mathscr{A}$. By a standard separation argument, there exists a nonzero $\psi \in \mathscr{X}^{\prime}$ such that $\psi(Y) \leq \psi\left(X+\lambda S_{T}\right)$ for any $\lambda \in \mathbb{R}$. Hence, we must have $\psi\left(S_{T}\right)=0$. Note that $\psi$ is positive due to Lemma 3.11. Since $S_{T}$ is strictly positive, $\psi\left(S_{T}\right)=0$ cannot hold and we conclude that $\rho_{\mathscr{A}, S}$ does not attain the value $-\infty$.

By Theorem 3.12 and $S$-additivity, to conclude the proof we just need to show that $\rho_{\mathscr{A}, S}(0)<\infty$. If this is not the case, then $\mathbb{R} S_{T} \cap \mathscr{A}=\emptyset$. Thus we can find a nonzero separating functional $\varphi \in \mathscr{X}^{\prime}$ such that $\lambda \varphi\left(S_{T}\right) \leq \varphi(X)$ for every $X \in \mathscr{A}$ and $\lambda \in \mathbb{R}$. This implies $\varphi\left(S_{T}\right)=0$, which is again in contrast to the positivity of $\varphi$ ensured by Lemma 3.11. Hence $\rho_{\mathscr{A}, S}(0)<\infty$, concluding the proof.

We now show that when the underlying acceptance set has nonempty interior, a convex risk measure which is finite-valued on a dense subspace is automatically finite-valued on the whole space. This is particularly useful when dealing with risk measures defined on $L^{p}, 1 \leq p<\infty$, or on Orlicz hearts $H^{\Phi}$, since it is typically not difficult to establish finiteness on the dense subspace $L^{\infty}$. The result is also valid for general convex maps whose domain has nonempty interior.

Proposition 3.15 Let $\mathscr{A} \subset \mathscr{X}$ be a convex acceptance set with nonempty interior and $S=\left(S_{0}, S_{T}\right)$ a traded asset. Assume $\rho_{\mathscr{A}, S}$ does not attain the value $-\infty$. If $\rho_{\mathscr{A}}, S$ is finite-valued on a dense linear subspace $\mathscr{S}$ of $\mathscr{X}$, then $\rho_{\mathscr{A}}, \mathrm{s}$ is finite-valued and continuous on $\mathscr{X}$.

Proof Assume $X \notin \operatorname{dom}\left(\rho_{\mathscr{A}}, S\right)$. Since the domain of $\rho_{\mathscr{A}, S}$ is convex and contains $\mathscr{A}$, by separation we find a nonzero $\psi \in \mathscr{X}^{\prime}$ such that $\psi(X) \leq \psi(Y)$ for all $Y \in \mathscr{S}$. But this implies $\psi$ must annihilate $\mathscr{S}$ and hence, by density, the whole space $\mathscr{X}$. Therefore, $\rho_{\mathscr{A}, S}$ must be finite-valued, hence continuous, on the whole of $\mathscr{X}$.

\subsection{Conic and coherent acceptance sets}

In this section, we focus our analysis on conic and coherent acceptance sets. We start with the main result characterizing the range of eligible assets for which a coherent risk measure is finite-valued, respectively continuous. We apply this result to risk measures based on TVaR-acceptability in $L^{p}$ spaces in Sect. 4.2. Note that if $\mathscr{A} \subset \mathscr{X}$ is a coherent acceptance set, the relation $X \leq \mathscr{A} Y$ defined by $Y-X \in \mathscr{A}$ is a preordering on $\mathscr{X}$ with positive cone $\mathscr{A}$. Note that $\leq \mathscr{A}$ is not an ordering unless $\mathscr{A}$ is pointed.

Theorem 3.16 Assume $\mathscr{A} \subset \mathscr{X}$ is a coherent acceptance set and let $S=\left(S_{0}, S_{T}\right)$ be a traded asset.

(i) The following statements are equivalent:

(a) $\rho_{\mathscr{A}, S}$ is finite-valued.

(b) $S_{T} \in \operatorname{core}(\mathscr{A})$.

(ii) The following statements are equivalent:

(a) $\rho_{\mathscr{A}, S}$ is continuous on $\mathscr{X}$. 
(b) $\rho_{\mathscr{A}}, S$ is continuous at 0 .

(c) $S_{T} \in \operatorname{int}(\mathscr{A})$.

Moreover, if $\mathscr{X}$ is an ordered normed space, then $\rho_{\mathscr{A}, S}$ is Lipschitz-continuous whenever $S_{T} \in \operatorname{int}(\mathscr{A})$.

Proof (i) Note that (b) is equivalent to $S_{T}$ being an order unit with respect to $\leq \mathscr{A}$. Hence, (b) implies (a) by Remark 3.2. To prove the converse, assume $\rho_{\mathscr{A}}, S$ is finitevalued but $S_{T} \notin \operatorname{core}(\mathscr{A})$. Then we can find $X \in \mathscr{X}$ such that $S_{T}+\lambda_{n} X \notin \mathscr{A}$ for a suitable sequence $\left(\lambda_{n}\right)$ of strictly positive numbers converging to zero. Equivalently, $X+\frac{1}{\lambda_{n}} S_{T} \notin \mathscr{A}$ for every $n \in \mathbb{N}$, implying $\rho_{\mathscr{A}, S}(X)=\infty$.

(ii) Clearly (a) implies (b). If $\rho_{\mathscr{A}, S}$ is continuous at 0 , then for $m>0 \geq \rho_{\mathscr{A}, S}(0)$, we have $\frac{m}{S_{0}} S_{T} \in \operatorname{int}(\mathscr{A})$ by Lemma 2.5 . Taking $m:=S_{0}$, we see that $S_{T} \in \operatorname{int}(\mathscr{A})$, proving that (b) implies (c). Finally, if (c) holds then we can again pass to the induced pre-ordering $\leq_{\mathscr{A}}$ and refer to Remark 3.2 to conclude the proof.

We conclude this section with a finiteness result in the context of conic acceptance sets, omitting the easy proof. This result will be of practical importance in the context of risk measures based on VaR-acceptability in $L^{p}$ spaces treated in Sect. 4.1.

Proposition 3.17 Assume $\mathscr{A} \subset \mathscr{X}$ is a conic acceptance set and let $S=\left(S_{0}, S_{T}\right)$ be a traded asset. The following statements hold:

(i) $\rho_{\mathscr{A}, S}<\infty$ if and only if $S_{T} \in \operatorname{core}(\mathscr{A})$.

(ii) $\rho_{\mathscr{A}, S}>-\infty$ if and only if $-S_{T} \in \operatorname{core}\left(\mathscr{A}^{c}\right)$.

In particular, if $\rho_{\mathscr{A}, S}$ is finite-valued, then core $(\mathscr{A})$ is nonempty.

\section{Applications}

We now apply our previous results to provide complete characterizations of finiteness and continuity for risk measures on $L^{p}$ spaces based on the two most prominent acceptability criteria in practice: value-at-risk and tail value-at-risk. We also provide a treatment of shortfall risk measures on Orlicz spaces arising from utility functions. Throughout this entire section, we maintain the assumption that $(\Omega, \mathcal{F}, \mathbb{P})$ is a nonatomic probability space.

\subsection{Acceptability based on value-at-risk}

In this subsection, we work in the setting of $\mathscr{X}=L^{p}$ for a fixed $0 \leq p<\infty$. The case $p=\infty$ is analogous to the case where $\mathscr{X}$ is the space of bounded measurable functions, for which we refer to [17].

For $\alpha \in(0,1)$, the value-at-risk of $X \in L^{p}$ at the level $\alpha$ is defined as

$$
\operatorname{VaR}_{\alpha}(X):=\inf \{m \in \mathbb{R}: \mathbb{P}(X+m<0) \leq \alpha\} .
$$

The set

$$
\mathscr{A}_{\alpha}:=\left\{X \in L^{p}: \operatorname{VaR}_{\alpha}(X) \leq 0\right\}=\left\{X \in L^{p}: \mathbb{P}(X<0) \leq \alpha\right\}
$$


is a conic acceptance set which is not convex and which is well known to be closed; see for instance Theorem 3 in [10]. The following lemma describes the interior of $\mathscr{A}_{\alpha}$.

Lemma 4.1 The acceptance set $\mathscr{A}_{\alpha}$ has nonempty interior in $L^{p}$. Moreover,

$$
\operatorname{int}\left(\mathscr{A}_{\alpha}\right)=\left\{X \in L^{p}: \mathbb{P}(X \leq 0)<\alpha\right\} .
$$

In particular, for $S_{T} \in L_{+}^{p}$, we have $S_{T} \in \operatorname{int}\left(\mathscr{A}_{\alpha}\right)$ if and only if $\mathbb{P}\left(S_{T}=0\right)<\alpha$.

Proof To prove (4.1), first recall that by Proposition 3.6, the core and the interior of any acceptance set in $L^{p}$ coincide. Take now $X \in L^{p}$ with $\mathbb{P}(X \leq 0)<\alpha$. If $X \notin \operatorname{core}\left(\mathscr{A}_{\alpha}\right)$, then we can find $Z \in L_{+}^{p}$ and $\lambda_{n} \downarrow 0$ such that $\mathbb{P}\left(X<\lambda_{n} Z\right)>\alpha$, implying $\mathbb{P}(X \leq 0) \geq \alpha$. But this contradicts what we assumed above; hence $X$ must belong to $\operatorname{core}\left(\mathscr{A}_{\alpha}\right)$.

To prove the converse inclusion, take $X \in \operatorname{core}\left(\mathscr{A}_{\alpha}\right)$ and assume $\mathbb{P}(X \leq 0) \geq \alpha$. Since $X \in \mathscr{A}_{\alpha}$, we have $\mathbb{P}(X>0)>0$ and thus $\mathbb{P}(0<X<\varepsilon)>0$ for some $\varepsilon>0$. Therefore, we find a sequence $\left(A_{n}\right)$ of pairwise disjoint measurable subsets of $\{0<X<\varepsilon\}$ with $0<\mathbb{P}\left(A_{n}\right)<n^{-p-2}$. Setting $Z:=1_{\{X \leq 0\}}+\sum_{n} n 1_{A_{n}} \in L_{+}^{p}$, it is easy to see that for every $\lambda>0$, there exists a positive integer $n$ for which $\mathbb{P}(X<\lambda Z) \geq \mathbb{P}(X \leq 0)+\mathbb{P}\left(A_{n}\right)>\alpha$. But this contradicts $X \in \operatorname{core}\left(\mathscr{A}_{\alpha}\right)$; hence (4.1) must hold.

Let $S=\left(S_{0}, S_{T}\right)$ be a traded asset. The corresponding risk measure based on VaRacceptability is

$$
\rho_{\mathscr{A}_{\alpha}, S}(X)=\inf \left\{m \in \mathbb{R}: \mathbb{P}\left(X+\frac{m}{S_{0}} S_{T}<0\right) \leq \alpha\right\} .
$$

The following proposition provides a characterization of the finiteness of $\rho_{\mathscr{A}_{\alpha}, S}$ and shows that risk measures based on VaR-acceptability can never be globally continuous, regardless of the choice of the eligible asset.

Proposition 4.2 Let $S=\left(S_{0}, S_{T}\right)$ be a traded asset. The following statements are equivalent:

(a) $\rho_{\mathscr{A}_{\alpha}, S}$ is finite-valued on $L^{p}$.

(b) $\mathbb{P}\left(S_{T}=0\right)<\min \{\alpha, 1-\alpha\}$.

Moreover, $\rho_{\mathscr{A}_{\alpha}, S}$ is never globally continuous on $L^{p}$.

Proof To characterize finiteness, by Proposition 3.17 and Lemma 4.1, we only need to show that $\rho_{\mathscr{A}_{\alpha}, S}$ never attains the value $-\infty$ if and only if $\mathbb{P}\left(S_{T}=0\right)<1-\alpha$. If $\mathbb{P}\left(S_{T}=0\right) \geq 1-\alpha$, then clearly $\rho_{\mathscr{A}_{\alpha}, S}(0)=-\infty$. For the converse, assume that $\mathbb{P}\left(S_{T}=0\right)<1-\alpha$. Because $\mathbb{P}\left(\left\{X<n S_{T}\right\} \cap\left\{S_{T}>0\right\}\right) \rightarrow \mathbb{P}\left(S_{T}>0\right)$ as $n \rightarrow \infty$ for any $X \in L^{P}$, we get $\mathbb{P}\left(X<n S_{T}\right)>\alpha$ for large enough $n \in \mathbb{N}$. Hence it follows that $\rho_{\mathscr{A}_{\alpha}, S}(X)>-\infty$.

To show that $\rho_{\mathscr{A}_{\alpha}, S}$ is never continuous on the whole $L^{p}$, take $\varepsilon>0$ and a measurable set $A$ with $\mathbb{P}(A)=\alpha$, and set $X:=-\left(S_{T}+\varepsilon\right) 1_{A} \in L^{p}$. Note that $\mathbb{P}(X<0)=\alpha$ 
and $\mathbb{P}\left(X+S_{T} \leq 0\right) \geq \alpha$. So $\rho_{\mathscr{A}_{\alpha}, S}(X) \leq 0<S_{0} \leq \rho_{\text {int }\left(\mathscr{A}_{\alpha}\right), S}(X)$, and Lemma 2.5 implies that $\rho_{\mathscr{A}_{\alpha}, S}$ cannot be (upper semi)continuous at $X$.

\subsection{Acceptability based on tail value-at-risk}

We continue to work on $\mathscr{X}=L^{p}$ for a fixed $1 \leq p<\infty$. As for value-at-risk, the case $p=\infty$ can be treated similarly to the case of bounded measurable functions which can be found in [17].

Fix $\alpha \in(0,1)$. The tail value-at-risk of $X \in L^{p}$ at the level $\alpha$ is defined as

$$
\operatorname{TVaR}_{\alpha}(X):=\frac{1}{\alpha} \int_{0}^{\alpha} \operatorname{VaR}_{\beta}(X) d \beta
$$

It is well known that $\mathrm{TVaR}_{\alpha}$ is cash additive and Lipschitz-continuous on $L^{1}$ and therefore also on $L^{p}$. Therefore, the set

$$
\mathscr{A}^{\alpha}:=\left\{X \in L^{p}: \operatorname{TVaR}_{\alpha}(X) \leq 0\right\}
$$

is a closed, coherent acceptance set which has nonempty interior. Moreover, note that $\mathscr{A}^{\alpha} \subset \mathscr{A}_{\alpha}$.

Lemma 4.3 The following holds:

$$
\operatorname{int}\left(\mathscr{A}^{\alpha}\right)=\left\{X \in L^{p}: \operatorname{TVaR}_{\alpha}(X)<0\right\} \subset\left\{X \in L^{p}: \mathbb{P}(X \leq 0)<\alpha\right\} .
$$

For $S_{T} \in L_{+}^{p}$, we have $S_{T} \in \operatorname{int}\left(\mathscr{A}^{\alpha}\right)$ if and only if $\mathbb{P}\left(S_{T}=0\right)<\alpha$.

Proof The equality in (4.2) follows from Remark 2.6. Moreover, since $\mathscr{A}^{\alpha} \subset \mathscr{A}_{\alpha}$, the inclusion in (4.2) is an immediate consequence of Lemma 4.1. Finally, if $X \in L_{+}^{p}$ and $\mathbb{P}(X=0)<\alpha$, we must find $\lambda>0$ in such a way that $\gamma:=\mathbb{P}(X<\lambda)<\alpha$. Then $\operatorname{VaR}_{\beta}(X)<0$ for all $\beta \in(\gamma, \alpha)$. Since $X$ is positive, this implies $\operatorname{TVaR}_{\alpha}(X)<0$.

Given a traded asset $S=\left(S_{0}, S_{T}\right)$, we consider the corresponding risk measure based on TVaR-acceptability, defined by

$$
\rho_{\mathscr{A}}^{\alpha}, S(X)=\inf \left\{m \in \mathbb{R}: \operatorname{TVaR}_{\alpha}\left(X+\frac{m}{S_{0}} S_{T}\right) \leq 0\right\} .
$$

The following proposition provides a characterization of the finiteness and continuity of TVaR-based risk measures and is a direct consequence of Lemma 4.3 and the results in Sect. 3.3. Note the strong contrast to VaR-based risk measures, which are never globally continuous on $L^{p}$ for $p<\infty$.

Proposition 4.4 Let $S=\left(S_{0}, S_{T}\right)$ be a traded asset. The following statements are equivalent: 
(a) $\rho_{\mathscr{A}^{\alpha}, S}$ is finite-valued on $L^{p}$.

(b) $\rho_{\mathscr{A} \alpha}^{\alpha}, S$ is Lipschitz-continuous on $L^{p}$.

(c) $\operatorname{TVaR}_{\alpha}\left(S_{T}\right)<0$.

(d) $\mathbb{P}\left(S_{T}=0\right)<\alpha$.

\subsection{Acceptability based on shortfall risk}

Cash-additive risk measures based on utility functions have been widely investigated on spaces of bounded measurable functions; see [19] for a general overview. As a means of unifying the treatment of utility maximization problems, Biagini and Frittelli proposed in [6] to work instead in the setting of Orlicz spaces; see also Biagini and Frittelli [7] and Arai [3].

Recall that a nonconstant function $u: \mathbb{R} \rightarrow \mathbb{R}$ is a utility function if it is concave and increasing. Note that this implies $u(-\infty):=\lim _{x \rightarrow-\infty} u(x)=-\infty$. The function defined by

$$
\widehat{u}(x):=u(0)-u(-|x|)
$$

is an Orlicz function in the sense of Definition 2.1.1 in [14]. By $H^{\widehat{u}}$ we denote the corresponding Orlicz heart associated with $(\Omega, \mathcal{F}, \mathbb{P})$.

We fix a level $\alpha \in \mathbb{R}$ such that $\alpha \leq u\left(x_{0}\right)$ for some $x_{0} \in \mathbb{R}$. Then the set

$$
\mathscr{A}_{u}:=\left\{X \in H^{\widehat{u}}: \mathbb{E}[u(X)] \geq \alpha\right\}
$$

is a convex acceptance set which in general is not coherent. Note that we disregard any level $\alpha$ strictly bounding $u$ from above, since then $\mathscr{A}_{u}$ would be empty. If $S=\left(S_{0}, S_{T}\right)$ is a traded asset, the corresponding shortfall risk measure on $H^{\widehat{u}}$ is defined by

$$
\rho_{\mathscr{A}_{u}, S}(X)=\inf \left\{m \in \mathbb{R}: \mathbb{E}\left[u\left(X+\frac{m}{S_{0}} S_{T}\right)\right] \geq \alpha\right\} .
$$

We start by describing the topological properties of the acceptance set $\mathscr{A}_{u}$.

\section{Lemma 4.5}

(i) The set $\mathscr{A}_{u}$ has nonempty interior if and only if $u\left(x_{0}\right)>\alpha$ for some $x_{0}>0$.

(ii) If $u$ is bounded from above, then $\mathscr{A}_{u}$ is closed.

Proof (i) To prove the "if" part, we show that $X:=x_{0} 1_{\Omega}$ is an interior point of $\mathscr{A}_{u}$. Choose $\lambda \in(0,1)$ in such a way that $\alpha-\lambda u\left(x_{0}\right)+(1-\lambda)(1-u(0)) \leq 0$. Note that for every $Y \in H^{\widehat{u}}$ with $\|Y\|_{\widehat{u}}<1-\lambda$, we have $\mathbb{E}\left[\widehat{u}\left(\frac{Y}{1-\lambda}\right)\right] \leq 1$, yielding

$$
\begin{aligned}
\mathbb{E}[-u(X+Y)] & \leq \lambda \mathbb{E}\left[-u\left(\frac{X}{\lambda}\right)\right]+(1-\lambda) \mathbb{E}\left[-u\left(\frac{Y}{1-\lambda}\right)\right] \\
& \leq-\lambda u\left(\frac{x_{0}}{\lambda}\right)+(1-\lambda) \mathbb{E}\left[\widehat{u}\left(\frac{Y}{1-\lambda}\right)\right]-(1-\lambda) u(0) \\
& \leq-\lambda u\left(x_{0}\right)+(1-\lambda)(1-u(0)) \\
& \leq-\alpha .
\end{aligned}
$$


As a result, $X+Y \in \mathscr{A}_{u}$ whenever $\|Y\|_{\widehat{u}}<1-\lambda$, showing that $X$ belongs to the interior of $\mathscr{A}_{u}$.

To prove the "only if" part, assume $u(x) \leq \alpha$ for all $x \in \mathbb{R}$. Fix $X \in \mathscr{A}_{u}$ and $r>0$. We claim that $Y \notin \mathscr{A}_{u}$ for some $Y \in H^{\widehat{u}}$ with $\|Y-X\|_{\widehat{u}} \leq r$. To this end, take $\gamma>0$ such that $\mathbb{P}(|X| \leq \gamma)>0$ and $\lambda>0$ for which $u(\gamma-\lambda)<\alpha$. Since $(\Omega, \mathcal{F}, \mathbb{P})$ is nonatomic, we can find a measurable set $A \subset\{|X| \leq \gamma\}$ satisfying $\widehat{u}\left(\frac{\lambda}{r}\right) \mathbb{P}(A) \leq 1$. Hence, setting $Y:=(X-\lambda) 1_{A}+X 1_{A^{c}}$, it follows that $\|Y-X\|_{\widehat{u}} \leq r$. Moreover, since $u(\gamma-\lambda)<\alpha$, we obtain

$$
\mathbb{E}[u(Y)] \leq u(\gamma-\lambda) \mathbb{P}(A)+\alpha \mathbb{P}\left(A^{c}\right)<\alpha,
$$

showing that $Y \notin \mathscr{A}_{u}$.

(ii) Assume $u$ is bounded from above and let $\left(X_{n}\right)$ be a sequence in $\mathscr{A}_{u}$ converging to $X$. Without loss of generality, we can assume $X_{n} \rightarrow X$ almost surely. Since $u$ is bounded from above, it follows from Fatou's lemma that $X \in \mathscr{A}_{u}$.

We can now provide a complete characterization of finiteness and continuity for risk measures based on shortfall risk on Orlicz hearts.

Proposition 4.6 Consider a traded asset $S=\left(S_{0}, S_{T}\right)$.

(i) If $u$ is bounded from above, the following statements are equivalent:

(a) $\rho_{\mathscr{A}_{u}}, S$ is finite-valued.

(b) $u\left(x_{0}\right)>\alpha$ for some $x_{0}>0$ and $\mathbb{P}\left(S_{T}=0\right)=0$.

In this case, $\rho_{\mathscr{A}_{u}}, \mathrm{~S}$ is continuous.

(ii) If $u$ is not bounded from above, then $\rho_{\mathscr{A}_{u}, S}$ is always finite-valued and continuous.

Proof (i) Assume (a) holds. Then $\mathscr{A}_{u}$ must have nonempty core by Proposition 3.8, and hence nonempty interior by Proposition 3.6. As a result, Lemma 4.5 implies $u\left(x_{0}\right)>\alpha$ for some $x_{0}>0$. Assume now that $\mathbb{P}\left(S_{T}=0\right)>0$. Since $u(-\infty)=-\infty$, taking $\xi>0$ large enough, we obtain for all $\lambda \in \mathbb{R}$

$$
\mathbb{E}\left[u\left(-\xi 1_{\Omega}+\lambda S_{T}\right)\right] \leq u(-\xi) \mathbb{P}\left(S_{T}=0\right)+\sup _{x \in \mathbb{R}} u(x) \mathbb{P}\left(S_{T}>0\right)<\alpha .
$$

As a result $\rho_{\mathscr{A}_{u}, S}\left(-\xi 1_{\Omega}\right)=\infty$, contradicting (a). Hence, (a) implies (b).

To prove the converse implication, assume (b) holds. Note that $\mathbb{P}\left(S_{T}=0\right)=0$ implies that $S_{T}$ is a strictly positive element in $H^{\widehat{u}}$. Moreover, $\mathscr{A}_{u}$ has nonempty interior by Lemma 4.5. Hence, (a) follows immediately from Corollary 3.14.

(ii) First, we show that $\rho_{\mathscr{A}_{u}, S}$ never attains $-\infty$. Indeed, assume $\rho_{\mathscr{A}_{u}, S}(X)=-\infty$ for some $X \in H^{\widehat{u}}$, and take $\beta<0$ such that

$$
\beta \mathbb{P}\left(S_{T}>0\right)+\mathbb{E}\left[u(X) 1_{\{u(X)>0\}}\right]<\alpha .
$$

Since $v:=u \vee \beta \geq u$, we have $\mathbb{E}\left[v\left(X-n S_{T}\right)\right] \geq \alpha$ for every positive integer $n$. Hence, using dominated convergence, it is easy to show that

$$
\beta \mathbb{P}\left(S_{T}>0\right)+\mathbb{E}\left[u(X) 1_{\{u(X)>0\}}\right] \geq \mathbb{E}\left[\beta 1_{\left\{S_{T}>0\right\}}+v(X) 1_{\left\{S_{T}=0\right\}}\right] \geq \alpha .
$$

But this contradicts (4.3), showing that $\rho_{\mathscr{A}_{u}, S}$ cannot attain the value $-\infty$. 
Now, since $u$ is not bounded from above, we always have $u\left(x_{0}\right)>\alpha$ for some $x_{0}>0$, hence the interior of $\mathscr{A}_{u}$ is nonempty by Lemma 4.5. Finally, take $\gamma>0$ so that $\mathbb{P}\left(S_{T}>\gamma\right)>0$. For any $\xi>0$ we can find $\lambda>0$ for which

$$
\mathbb{E}\left[u\left(-\xi 1_{\Omega}+\lambda S_{T}\right)\right] \geq u(-\xi) \mathbb{P}\left(S_{T} \leq \gamma\right)+u(-\xi+\lambda \gamma) \mathbb{P}\left(S_{T}>\gamma\right) \geq \alpha,
$$

showing that $\rho_{\mathscr{A}_{u}, S}\left(-\xi 1_{\Omega}\right)<\infty$. Since $1_{\Omega}$ is a strictly positive element, we can apply Theorem 3.12 to find that $\rho_{\mathscr{A}_{u}, S}$ is finite-valued and continuous, concluding the proof.

Note that Lemma 4.5 continues to hold if the underlying reference space is taken to be the Orlicz space $L^{\widehat{u}}$. Our next example shows that when $u$ is the exponential utility function, the risk measure $\rho_{\mathscr{A}}, S$ is never finite-valued on $L^{\widehat{u}}$ even though its domain has nonempty interior. This extends the cash-additive example by Biagini and Frittelli at the end of Sect. 5.1 in [7], which was used to highlight that the results in Cheridito and $\mathrm{Li} \mathrm{[11]} \mathrm{for} \mathrm{Orlicz} \mathrm{hearts} \mathrm{are} \mathrm{not} \mathrm{valid} \mathrm{in} \mathrm{the} \mathrm{context} \mathrm{of} \mathrm{general} \mathrm{Orlicz}$ spaces.

Example 4.7 Let $u(x):=1-e^{-x}$ be the exponential utility function. For any traded asset $S=\left(S_{0}, S_{T}\right)$, the risk measure $\rho_{\mathscr{A}_{u}, S}$ is not finite-valued on $L^{\widehat{u}}$. Indeed, since $(\Omega, \mathcal{F}, \mathbb{P})$ is nonatomic, we can always find $Y \in L^{1 / 2} \backslash L^{1}$ such that $Y \geq 1$ almost surely and $Y$ is independent of $S_{T}$. Setting $X:=-\log Y$, it is easy to see that $X \in L^{\widehat{u}}$ and $\mathbb{E}\left[e^{-X}\right]=\infty$. As a consequence, for any $\lambda \in \mathbb{R}$ we have

$$
\mathbb{E}\left[u\left(X+\lambda S_{T}\right)\right]=1-\mathbb{E}\left[e^{-X}\right] \mathbb{E}\left[e^{-\lambda S_{T}}\right]=-\infty<\alpha,
$$

showing that $\rho_{\mathscr{A}_{u}, S}(X)=\infty$.

Remark 4.8 (i) The previous example has an interesting consequence. Since $\mathscr{A}_{u}$ is convex and has nonempty interior in $L^{\widehat{u}}$, Corollary 3.14 implies that the Orlicz space $L^{\widehat{u}}$ has no strictly positive elements. We do not know whether, more generally, whenever a nontrivial Orlicz heart and the corresponding Orlicz space do not coincide, the Orlicz space does not possess strictly positive elements.

(ii) Note that $1_{\Omega}$ is a strictly positive element in every nontrivial Orlicz heart but not in a general Orlicz space, unless the two coincide. This is the fundamental reason why the results in Cheridito and $\mathrm{Li}$ [11] on Orlicz hearts are not always applicable in the context of general Orlicz spaces.

\section{Cash subadditivity and quasiconvexity}

This final section is devoted to discussing the link between the general risk measures studied in this paper and cash-subadditive as well as quasiconvex risk measures. Establishing this link is important since our framework provides a natural setting to deal with a defaultable reference asset, and cash subadditivity was introduced in [16] to address the possible defaultability of the given reference bond. Moreover, quasiconvexity arises naturally in the presence of a convex acceptability criterion when the reference asset is not liquidly traded. 


\subsection{Cash subadditivity and defaultable assets}

In the recent influential paper [16], El Karoui and Ravanelli questioned the axiom of cash additivity and introduced the new class of (convex) cash-subadditive risk measures on $L^{\infty}$ in order to "model stochastic and/or ambiguous interest rates or defaultable contingent claims". In a more general setting, i.e., working in $L^{p}, 0 \leq p \leq \infty$, and without requiring finiteness or convexity, a cash-subadditive risk measure is defined as a decreasing function $\rho: L^{p} \rightarrow \overline{\mathbb{R}}$ satisfying

$$
\rho\left(X+\lambda 1_{\Omega}\right) \geq \rho(X)-\lambda \text { for all } \lambda>0 \text { and } X \in L^{p} .
$$

Consider an acceptance set $\mathscr{A} \subset L^{p}$ and a traded asset $S=\left(S_{0}, S_{T}\right)$. Note that in the framework considered thus far, the $S$-additivity of $\rho_{\mathscr{A}, S}$ is a direct consequence of the fact that the price of $\lambda$ units of $S$ is $\lambda S_{0}$. Hence, unless we assume a nonlinear pricing rule as we do in the final section of this paper, $\rho_{\mathscr{A}}, S$ will always be $S$-additive. Consequently, cash subadditivity is not a surrogate for $S$-additivity, but rather a property $\rho_{\mathscr{A}, S}$ may or may not have. If we wish to interpret risk measures as capital requirements which measure the distance of future financial positions to acceptability, any new property stipulated for risk measures, such as cash subadditivity, needs to be justified by a corresponding financially meaningful property of either $\mathscr{A}$ or $S$. Therefore, in this section we investigate what makes $\rho_{\mathscr{A}, S}$ cash subadditive. By doing so, we also provide a better financial insight into the axiom of cash subadditivity. In particular, our results show that this assumption is typically not satisfied when the asset $S$ is defaultable, thus raising questions at least about part of the interpretation given in [16].

Note that, by $S$-additivity, cash subadditivity of $\rho_{\mathscr{A}, S}$ is equivalent to

$$
\rho_{\mathscr{A}, S}\left(X+\lambda 1_{\Omega}\right) \geq \rho_{\mathscr{A}, S}\left(X+\frac{\lambda}{S_{0}} S_{T}\right) \text { for all } \lambda>0 \text { and } X \in L^{p} \text {. }
$$

Assume $S=\left(S_{0}, S_{T}\right)$ is a defaultable bond with face value 1 , recovery rate $0 \leq S_{T} \leq 1$, and price $S_{0}<1$. Then we can interpret $S_{0}$ as the invested capital and $1-S_{0}$ as the interest payment. The following result shows that if the invested capital is not at risk, i.e., if the bond can only default on the interest payment, then the risk measure $\rho_{\mathscr{A}, S}$ is always cash subadditive.

Proposition 5.1 Let $\mathscr{A} \subset L^{p}$ be an acceptance set and $S=\left(S_{0}, S_{T}\right)$ a traded asset. Assume $\mathbb{P}\left(S_{T}<S_{0}\right)=0$. Then $\rho_{\mathscr{A}, S}$ is cash subadditive.

Proof Taking $X \in L^{p}$ and $\lambda>0$ and noting that $\lambda 1_{\Omega} \leq \frac{\lambda}{S_{0}} S_{T}$, we immediately obtain by (5.1) and monotonicity that $\rho_{\mathscr{A}, S}$ is cash subadditive.

We investigate now the case where the capital invested in the asset $S=\left(S_{0}, S_{T}\right)$ is at risk, i.e., when $\mathbb{P}\left(S_{T}<S_{0}\right)>0$. In this situation, we shall see that cash subadditivity is typically not satisfied. Moreover, cash subadditivity turns out to depend not only on the payoff $S_{T}$ of the eligible asset, but also on the prevailing price $S_{0}$. As 
such, this property is not stable with respect to changes in the price of the eligible asset, a circumstance which would seem to limit its practical usefulness.

We start by providing a necessary condition for cash subadditivity for a general underlying acceptance set, and a sufficient condition in the coherent case.

Proposition 5.2 Let $\mathscr{A} \subset L^{p}$ be an acceptance set containing 0 and $S=\left(S_{0}, S_{T}\right)$ a traded asset. The following statements hold:

(i) If $\rho_{\mathscr{A}, S}$ is cash subadditive, then $S_{T}-S_{0} 1_{\Omega} \in \overline{\mathscr{A}}$.

(ii) If $\mathscr{A}$ is coherent and $S_{T}-S_{0} 1_{\Omega} \in \mathscr{A}$, then $\rho_{\mathscr{A}, S}$ is cash subadditive.

In particular, if $\mathscr{A}$ is closed and coherent, then $\rho_{\mathscr{A}, S}$ is cash subadditive if and only if $S_{T}-S_{0} 1_{\Omega} \in \mathscr{A}$.

Proof To prove (i), assume cash subadditivity and note that taking $X:=-S_{0} 1_{\Omega}$ and $\lambda:=S_{0}$ in $(5.1)$, we obtain

$$
\rho_{\mathscr{A}, S}\left(S_{T}-S_{0} 1_{\Omega}\right)=\rho_{\mathscr{A}, S}\left(X+\frac{\lambda}{S_{0}} S_{T}\right) \leq \rho_{\mathscr{A}, S}\left(X+\lambda 1_{\Omega}\right)=\rho_{\mathscr{A}, S}(0) \leq 0 .
$$

Hence, $S_{T}-S_{0} 1_{\Omega} \in \overline{\mathscr{A}}$.

To prove (ii), assume $\rho_{\mathscr{A}, S}$ is not cash subadditive. Then we find $X \in L^{p}$ and $\lambda>0$ for which

$$
\rho_{\mathscr{A}, S}\left(X+\lambda 1_{\Omega}\right)<0<\rho_{\mathscr{A}, S}\left(X+\frac{\lambda}{S_{0}} S_{T}\right)
$$

so that $X+\lambda 1_{\Omega} \in \mathscr{A}$ while $X+\frac{\lambda}{S_{0}} S_{T} \notin \mathscr{A}$. Since the set $\mathscr{A}$ is coherent and $X+\frac{\lambda}{S_{0}} S_{T}=X+\lambda 1_{\Omega}+\frac{\lambda}{S_{0}}\left(S_{T}-S_{0} 1_{\Omega}\right)$, we conclude that $S_{T}-S_{0} 1_{\Omega} \notin \mathscr{A}$. This shows that (ii) holds.

The following corollaries provide a characterization of cash subadditivity for risk measures based on TVaR-acceptability and scenario-based acceptability, respectively. In particular, for TVaR-acceptability at level $\alpha$, it turns out that the corresponding risk measure $\rho_{\mathscr{A} \alpha} \alpha$ is not cash subadditive whenever the probability that the invested capital is at risk exceeds the level $\alpha$.

Corollary 5.3 (Tail value-at-risk) Let $\mathscr{A}^{\alpha} \subset L^{p}$ be the acceptance set based on tail value-at-risk at level $\alpha \in(0,1)$, and let $S=\left(S_{0}, S_{T}\right)$ be a traded asset. Then $\rho_{\mathscr{A}} \alpha, S$ is cash subadditive if and only if $\operatorname{TVaR}_{\alpha}\left(S_{T}\right) \leq-S_{0}$.

In particular, if $\rho_{\mathscr{A} \alpha}^{\alpha}, S$ is cash subadditive, then $\mathbb{P}\left(S_{T}<S_{0}\right) \leq \alpha$.

Corollary 5.4 (Scenarios) Let $A \in \mathcal{F}$ and define $\mathscr{A}(A):=\left\{X \in L^{p}: X 1_{A} \geq 0\right\}$. If $S=\left(S_{0}, S_{T}\right)$ is a traded asset, then $\rho_{\mathscr{A}}(A), S$ is cash subadditive if and only if $\mathbb{P}\left(A \cap\left\{S_{T}<S_{0}\right\}\right)=0$.

The following result shows that VaR-based risk measures are never cash subadditive as soon as the invested capital is at risk. 
Proposition 5.5 (Value-at-risk) Let $\mathscr{A}_{\alpha} \subset L^{p}$ be the acceptance set based on valueat-risk at level $\alpha \in(0,1)$, and $S=\left(S_{0}, S_{T}\right)$ a traded asset. Then $\rho_{\mathscr{A}_{\alpha}, S}$ is cash subadditive if and only if $\mathbb{P}\left(S_{T}<S_{0}\right)=0$.

Proof The "if" part follows from Proposition 5.1. To prove the "only if" part, assume $\rho_{\mathscr{A}_{\alpha}, S}$ is cash subadditive but $\mathbb{P}\left(S_{T}<S_{0}\right)>0$. Take $\varepsilon \in(0,1)$ such that $\mathbb{P}\left(S_{T} \leq \varepsilon S_{0}\right)>0$. Since $\mathbb{P}\left(S_{T}<S_{0}\right) \leq \alpha$ by part (i) in Proposition 5.2, we can find $0<\delta<\mathbb{P}\left(S_{T} \leq \varepsilon S_{0}\right)$ satisfying $\mathbb{P}\left(S_{T} \geq S_{0}\right)>\alpha+\delta-\mathbb{P}\left(S_{T}<S_{0}\right)>0$. Moreover, since $(\Omega, \mathcal{F}, \mathbb{P})$ is nonatomic, we find a measurable subset $A$ of $\left\{S_{T} \geq S_{0}\right\}$ such that $\mathbb{P}(A)=\alpha+\delta-\mathbb{P}\left(S_{T}<S_{0}\right)$. Take $\gamma>0$ such that $(1+\gamma) \varepsilon<1$ and set

$$
X:= \begin{cases}-1 & \text { on } B, \\ -\frac{2+\gamma}{S_{0}} S_{T} & \text { on } B^{c},\end{cases}
$$

where $B:=\left\{S_{T} \leq \varepsilon S_{0}\right\} \cup\left(\left\{S_{T} \geq S_{0}\right\} \backslash A\right)$. Then $\mathbb{P}\left(X+1_{\Omega}<0\right) \leq \mathbb{P}\left(B^{c}\right)<\alpha$, implying $\rho_{\mathscr{A}_{\alpha}, S}\left(X+1_{\Omega}\right) \leq 0$. Moreover,

$$
X+\frac{1}{S_{0}} S_{T}+\frac{\gamma}{S_{0}} S_{T} \leq-1+(1+\gamma) \varepsilon<0 \quad \text { on }\left\{S_{T} \leq \varepsilon S_{0}\right\}
$$

and

$$
X+\frac{1}{S_{0}} S_{T}+\frac{\gamma}{S_{0}} S_{T}=-\frac{1}{S_{0}} S_{T}<0 \quad \text { on } B^{c} .
$$

Hence, it follows that

$$
\mathbb{P}\left(X+\frac{1}{S_{0}} S_{T}+\frac{\gamma}{S_{0}} S_{T}<0\right) \geq \mathbb{P}\left(S_{T}<S_{0}\right)+\mathbb{P}(A)=\alpha+\delta>\alpha,
$$

showing that $\rho_{\mathscr{A}_{\alpha}, S}\left(X+\frac{1}{S_{0}} S_{T}\right) \geq \gamma>0$. But since we have already proved that $\rho_{\mathscr{A}_{\alpha}, S}\left(X+1_{\Omega}\right) \leq 0$, we conclude by (5.1) that $\rho_{\mathscr{A}_{\alpha}, S}$ cannot be cash subadditive, contradicting our initial assumption.

Remark 5.6 Proposition 5.5 shows that Proposition 5.2 fails if we drop the assumption of convexity. In fact, the assumption of conicity cannot be dropped either. Indeed, consider $\mathscr{A}:=\left\{X \in L^{p}: \mathbb{E}[X] \geq \alpha\right\}$ for a fixed $\alpha \in \mathbb{R}$, and let $S=\left(S_{0}, S_{T}\right)$ be a traded asset. Then it is easy to see that $\rho_{\mathscr{A}, S}$ is cash subadditive if and only if $\mathbb{E}\left[S_{T}\right] \geq S_{0}$.

\subsection{Quasiconvexity and illiquid eligible assets}

We now proceed to extend in a natural way the definition of a risk measure to account for situations where the eligible asset is not liquidly traded. We assume $\mathscr{X}$ is a general ordered topological vector space.

Thus far we have dealt with liquidly traded assets $S=\left(S_{0}, S_{T}\right)$ for which the price of $\lambda \in \mathbb{R}$ units of $S$ is $\pi(\lambda):=\lambda S_{0}$. When the asset is not liquidly traded, the pricing functional $\pi: \mathbb{R} \rightarrow \mathbb{R}$ will no longer be linear. Here, we only assume $\pi$ is strictly 
increasing and satisfies $\pi(0)=0$ and $\pi(1)=S_{0}$. Note that we do not require any form of continuity for $\pi$, thus allowing price jumps, which are a typical feature of an illiquid market.

Definition 5.7 Let $\mathscr{A} \subset \mathscr{X}$ be an acceptance set and $S=\left(S_{0}, S_{T}\right)$ a traded asset with pricing functional $\pi$. The risk measure with respect to $\mathscr{A}, S$ and $\pi$ is the function $\rho_{\mathscr{A}, S, \pi}: \mathscr{X} \rightarrow \overline{\mathbb{R}}$ defined by

$$
\rho_{\mathscr{A}, S, \pi}(X):=\inf \left\{\pi(\lambda): \lambda \in \mathbb{R} \text { with } X+\lambda S_{T} \in \mathscr{A}\right\} .
$$

From now on we assume that $\mathscr{A}$ is closed. Then we can reduce risk measures with respect to illiquid eligible assets to risk measures of the form $\rho_{\mathscr{A}, S}$. Note that even if the asset $S$ is not liquidly traded, we still write $\rho_{\mathscr{A}, S}$ to denote the risk measure we should get if we assumed full liquidity. As usual we set $\pi( \pm \infty):=\lim _{\lambda \rightarrow \pm \infty} \pi(\lambda)$.

Lemma 5.8 Let $\mathscr{A} \subset \mathscr{X}$ be a closed acceptance set and $S=\left(S_{0}, S_{T}\right)$ a traded asset with pricing functional $\pi$. Then for all $X \in \mathscr{X}$,

$$
\rho_{\mathscr{A}, S, \pi}(X)=\pi\left(\frac{1}{S_{0}} \rho_{\mathscr{A}, S}(X)\right) .
$$

Proof Take $X \in \mathscr{X}$. Since $\mathscr{A}$ is closed, the set of all $\lambda \in \mathbb{R}$ satisfying $X+\lambda S_{T} \in \mathscr{A}$ is a closed interval, possibly the full real line. As a result of the monotonicity of $\pi$, the equality (5.2) follows.

Remark 5.9 (i) Note that since $\mathscr{A}$ is closed, $\rho_{\mathscr{A}, S}$ is lower semicontinuous by Remark 2.6. However, this need not be the case for $\rho_{\mathscr{A}, S, \pi}$ unless $\pi$ is left continuous.

(ii) Lemma 5.8 should be compared with Example 2.2 in [9], where the payoff of the reference asset is $S_{T}=1_{\Omega}$. There, formula (5.2) is obtained by requiring the upper semicontinuity of $\pi$ rather than the closedness of $\mathscr{A}$.

The property of quasiconvexity is known to be the minimal property a risk measure needs to have to capture diversification effects. Quasiconvexity of risk measures has been extensively studied for instance in Cerreia-Vioglio et al. [9] and Drapeau and Kupper [13]. Recall that a function $\rho: \mathscr{X} \rightarrow \overline{\mathbb{R}}$ is called quasiconvex if the level sets $\{X \in \mathscr{X}: \rho(X) \leq \alpha\}$ are convex for every $\alpha \in \mathbb{R}$. As for the cash-additive case, it is easy to see that for an $S$-additive risk measure, quasiconvexity is equivalent to convexity. Hence, genuine quasiconvexity can only be observed if the pricing rule for $S$ is not linear. The next proposition provides a characterization of quasiconvex risk measures of the form $\rho_{\mathscr{A}, S, \pi}$.

Proposition 5.10 Let $\mathscr{A} \subset \mathscr{X}$ be a closed acceptance set and $S=\left(S_{0}, S_{T}\right)$ a traded asset with pricing functional $\pi$. Then $\rho_{\mathscr{A}, S, \pi}$ is quasiconvex if and only if $\mathscr{A}$ is convex. 
Proof Assume $\mathscr{A}$ is convex. Being the composition of a convex and an increasing function by (5.2), the map $\rho_{\mathscr{A}, S, \pi}$ is quasiconvex. On the other hand, if $\rho_{\mathscr{A}, S, \pi}$ is quasiconvex, then the set $\mathscr{B}:=\left\{X \in \mathscr{X}: \rho_{\mathscr{A}, S, \pi}(X) \leq 0\right\}$ is convex. Clearly, $\mathscr{A} \subset \mathscr{B}$. Take $X \in \mathscr{B}$ and note that $\pi\left(\frac{1}{S_{0}} \rho_{\mathscr{A}, S}(X)\right)=\rho_{\mathscr{A}, S, \pi}(X) \leq 0$. Since $\pi(0)=0$ and $\pi$ is strictly increasing, we immediately obtain $\rho_{\mathscr{A}, S}(X) \leq 0$. But this implies that $X \in \mathscr{A}$ as a consequence of Lemma 2.5 .

We now provide a dual representation for quasiconvex risk measures of the form $\rho_{\mathscr{A}, S, \pi}$. In contrast to Proposition 5 in Drapeau and Kupper [13], we do not require lower semicontinuity but exploit the special structure of $\rho_{\mathscr{A}}, S, \pi$. In particular, our representation formula (5.3) below allows a transparent interpretation in terms of the fundamental financial primitives: the acceptance set, the eligible asset, and the pricing functional. Recall that the Fenchel conjugate of a map $\rho: \mathscr{X} \rightarrow \overline{\mathbb{R}}$ is the function $\rho^{*}: \mathscr{X}^{\prime} \rightarrow \overline{\mathbb{R}}$ defined by

$$
\rho^{*}(\psi):=\sup _{X \in \mathscr{X}}\{\psi(X)-\rho(X)\}
$$

Moreover, for a traded asset $S=\left(S_{0}, S_{T}\right)$, we introduce the set

$$
\mathscr{X}_{+, S}^{\prime}:=\left\{\psi \in \mathscr{X}_{+}^{\prime}: \psi\left(S_{T}\right)=S_{0}\right\}
$$

Proposition 5.11 Assume $\mathscr{X}$ is locally convex. Let $\mathscr{A} \subset \mathscr{X}$ be a closed, convex acceptance set and $S=\left(S_{0}, S_{T}\right)$ a traded asset with pricing functional $\pi$. If $\rho_{\mathscr{A}, S}$ is continuous at an interior point $X$ of its domain of finiteness, then

$$
\rho_{\mathscr{A}, S, \pi}(X)=\max _{\psi \in \mathscr{X}_{+, S}^{\prime}}\left\{\pi\left(\frac{\psi(-X)-\rho_{\mathscr{A}, S}^{*}(-\psi)}{S_{0}}\right)\right\} .
$$

Proof Being finite at $X$, the convex and lower semicontinuous map $\rho_{\mathscr{A}, S}$ cannot attain the value $-\infty$ by Proposition 2.4 in [15]. Then, following the lines of the proof of Corollary 7 in [20], we obtain the standard dual representation

$$
\rho_{\mathscr{A}, S}(X)=\sup _{\psi \in \mathscr{X}_{+, S}^{\prime}}\left\{\psi(-X)-\rho_{\mathscr{A}, S}^{*}(-\psi)\right\} .
$$

Now take $m>\rho_{\mathscr{A}, S}(X)$. Since $\rho_{\mathscr{A}, S}$ is continuous at $X$, the interior of $\mathscr{A}$ is nonempty and $X_{m}:=X+\frac{m}{S_{0}} S_{T} \in \operatorname{int}(\mathscr{A})$ by Lemma 2.5 . Note that we have $\tilde{X}:=X+\frac{\rho_{\mathscr{A}, S}(X)}{S_{0}} S_{T} \in \partial \mathscr{A}$. As a result, Lemma 7.7 in [1] implies that $\tilde{X}$ is a support point of $\mathscr{A}$. Hence,

$$
\varphi(\tilde{X})=\inf _{Z \in \mathscr{A}} \varphi(Z)
$$

for some nonzero functional $\varphi \in \mathscr{X}^{\prime}$, which is positive by Lemma 3.11. Moreover, we must have $\varphi\left(S_{T}\right)>0$ since otherwise $\varphi\left(X_{m}\right)=\inf _{Y \in \mathscr{A}} \varphi(Y)$ which is not possible because $X_{m} \in \operatorname{int}(\mathscr{A})$. Rescaling $\varphi$ we may assume that $\varphi\left(S_{T}\right)=S_{0}$. Finally, 
taking $Y \in \operatorname{dom}\left(\rho_{\mathscr{A}, S}\right)$, we can conclude that

$\varphi(X)+\rho_{\mathscr{A}, S}(X)=\varphi(\widetilde{X})=\inf _{Z \in \mathscr{A}} \varphi(Z) \leq \varphi\left(Y+\frac{\rho_{\mathscr{A}, S}(Y)}{S_{0}} S_{T}\right)=\varphi(Y)+\rho_{\mathscr{A}, S}(Y)$.

Hence, the supremum in (5.4) is attained at $\varphi$ so that (5.3) now easily follows from Lemma 5.8 and the monotonicity of $\pi$.

Remark 5.12 Note that our attainability result is not implied by Theorem 1 in [7] since we do not assume $\mathscr{X}$ is a Frechét lattice. In particular, it remains valid on $L^{p}$ spaces when equipped with any weak topology, for instance on $L^{\infty}$ endowed with the weak* topology $\sigma\left(L^{\infty}, L^{1}\right)$.

We now assume $\mathscr{X}=L^{p}$ over a fixed probability space $(\Omega, \mathcal{F}, \mathbb{P})$. In [9] it is suggested that cash subadditivity may arise when the reference (risk-free) asset is illiquidly traded. Since the most natural way to incorporate illiquidity is by considering nonlinear pricing functionals as above, it is interesting to see whether our risk measures $\rho_{\mathscr{A}}, S, \pi$ are cash subadditive. The following result shows that in the common situations, this can only be true if the pricing functional $\pi$ is continuous, thus ruling out examples of illiquid markets where the pricing rule may have jumps.

Proposition 5.13 Fix $1 \leq p \leq \infty$ and let $\mathscr{A} \subset L^{p}$ be a closed, convex acceptance set with nonempty interior. Consider a traded asset $S=\left(S_{0}, S_{T}\right)$ with pricing functional $\pi$. If $\rho_{\mathscr{A}, S, \pi}$ is cash subadditive and $\rho_{\mathscr{A}, S}(0) \in \mathbb{R}$, then $\pi$ is continuous.

Proof We first prove that for every $\lambda>0$, we have

$$
-\infty<\rho_{\mathscr{A}, S}\left(\lambda 1_{\Omega}\right)<\rho_{\mathscr{A}, S}(0) .
$$

Fix $\lambda>0$ and note that since it is convex and lower semicontinuous, the risk measure $\rho_{\mathscr{A}}, S$ cannot assume the value $-\infty$ by Proposition 2.4 in [15]. Moreover, $X:=\frac{\rho_{\mathscr{A}, S}(0)}{S_{0}} S_{T}$ belongs to $\partial \mathscr{A}$ so that $X+\lambda 1_{\Omega} \in \operatorname{int}(\mathscr{A})$. Indeed, assume to the contrary that $X+\lambda 1_{\Omega} \in \partial \mathscr{A}$. Since $X$ is a support point of $\mathscr{A}$ by Lemma 7.7 in [1], we find a nonzero $\psi \in \mathscr{X}^{\prime}$ with $\psi\left(X+\lambda 1_{\Omega}\right) \leq \psi(Y)$ for all $Y \in \mathscr{A}$. In particular, choosing $Y:=X$, we get $\psi\left(1_{\Omega}\right) \leq 0$ which is impossible because $1_{\Omega}$ is a strictly positive element in $L^{p}$ and $\psi$ must be positive by Lemma 3.11. In conclusion, we obtain

$$
\rho_{\mathscr{A}, S}\left(\lambda 1_{\Omega}\right)-\rho_{\mathscr{A}, S}(0)=\rho_{\mathscr{A}, S}\left(X+\lambda 1_{\Omega}\right)<0
$$

proving (5.5).

Now assume that $\pi$ is not left-continuous at $x_{0} \in \mathbb{R}$ and denote the size of the jump by $\gamma:=\pi\left(x_{0}\right)-\lim _{x \uparrow x_{0}} \pi(x)>0$. Take $\xi \in \mathbb{R}$ such that, setting $X:=\xi S_{T}$, we have $\rho_{\mathscr{A}, S}(X)=\rho_{\mathscr{A}, S}(0)-\xi S_{0}=x_{0} S_{0}$. Hence, $\rho_{\mathscr{A}, S}\left(X+\lambda 1_{\Omega}\right)=\rho_{\mathscr{A}, S}\left(\lambda 1_{\Omega}\right)-\xi S_{0}<$ $x_{0} S_{0}$ for any $\lambda>0$ by (5.5). But then for $\lambda \in(0, \gamma)$, we get

$$
\rho_{\mathscr{A}, S, \pi}(X)-\rho_{\mathscr{A}, S, \pi}\left(X+\lambda 1_{\Omega}\right)=\pi\left(x_{0}\right)-\pi\left(\frac{\rho_{\mathscr{A}, S}\left(X+\lambda 1_{\Omega}\right)}{S_{0}}\right) \geq \gamma>\lambda
$$

showing that $\rho_{\mathscr{A}, S, \pi}$ is not cash subadditive. 
Similarly, assume that $\pi$ is not right-continuous at $x_{0} \in \mathbb{R}$ and define now $\gamma:=\lim _{x \downarrow x_{0}} \pi(x)-\pi\left(x_{0}\right)>0$. For $\lambda \in(0, \gamma)$, we find $\xi \in \mathbb{R}$ such that, setting $X:=\xi S_{T}$, we have $\rho_{\mathscr{A}, S}\left(X+\lambda 1_{\Omega}\right)=\rho_{\mathscr{A}, S}\left(\lambda 1_{\Omega}\right)-\xi S_{0}=x_{0} S_{0}$. Because (5.5) gives $\rho_{\mathscr{A}, S}(X)=\rho_{\mathscr{A}, S}(0)-\xi S_{0}>x_{0} S_{0}$, we conclude that

$$
\rho_{\mathscr{A}, S, \pi}(X)-\rho_{\mathscr{A}, S, \pi}\left(X+\lambda 1_{\Omega}\right)=\pi\left(\frac{\rho_{\mathscr{A}, S}(X)}{S_{0}}\right)-\pi\left(x_{0}\right) \geq \gamma>\lambda
$$

Again, this implies that $\rho_{\mathscr{A}, S, \pi}$ is not cash subadditive. In conclusion, for $\rho_{\mathscr{A}, S, \pi}$ to be cash subadditive, the pricing functional must be continuous.

Acknowledgements Partial support through the SNF project 51NF40-144611 "Capital adequacy, valuation, and portfolio selection for insurance companies" is gratefully acknowledged. Financial support by the National Centre of Competence in Research "Financial Valuation and Risk Management" (NCCR FinRisk), project "Mathematical Methods in Financial Risk Management", is gratefully acknowledged by W. Farkas and C. Munari. Part of this research was undertaken while P. Koch-Medina was employed by Swiss Re.

We gratefully acknowledge the careful review and constructive feedback provided by two anonymous referees. In particular, part of the proof of Proposition 4.6 could be significantly shortened following an idea of one of them.

\section{References}

1. Aliprantis, Ch.D., Border, K.C.: Infinite Dimensional Analysis: A Hitchhiker's Guide, 3rd edn. Springer, Berlin (2006)

2. Aliprantis, Ch.D., Tourky, R.: Cones and Duality. American Mathematical Society, Providence (2007)

3. Arai, T.: Good deal bounds induced by shortfall risk. SIAM J. Financ. Math. 2, 1-21 (2011)

4. Artzner, Ph., Delbaen, F., Eber, J.-M., Heath, D.: Coherent measures of risk. Math. Finance 9, 203228 (1999)

5. Artzner, Ph., Delbaen, F., Koch-Medina, P.: Risk measures and efficient use of capital. ASTIN Bull. 39, 101-116 (2009)

6. Biagini, S., Frittelli, M.: A unified framework for utility maximization problems: an Orlicz space approach. Ann. Appl. Probab. 18, 929-966 (2008)

7. Biagini, S., Frittelli, M.: On the extension of the Namioka-Klee theorem and on the Fatou property for risk measures. In: Delbaen, F., Rasonyi, M., Stricker, C. (eds.) Optimality and Risk: Modern Trends in Mathematical Finance, pp. 1-28. Springer, Berlin (2009)

8. Borwein, J.M.: Automatic continuity and openness of convex relations. Proc. Am. Math. Soc. 99, 49-55 (1987)

9. Cerreia-Vioglio, S., Maccheroni, F., Marinacci, M., Montrucchio, L.: Risk measures: rationality and diversification. Math. Finance 21, 743-774 (2011)

10. Chambers, C.P.: An axiomatization of quantiles on the domain of distribution functions. Math. Finance 19, 335-342 (2009)

11. Cheridito, P., Li, T.: Risk measures on Orlicz hearts. Math. Finance 19, 184-214 (2009)

12. Delbaen, F.: Coherent risk measures on general probability spaces. In: Sandmann, K., Schönbucher, P.J. (eds.) Advances in Finance and Stochastics: Essays in Honour of Dieter Sondermann, pp. 1-37. Springer, Berlin (2002)

13. Drapeau, S., Kupper, M.: Risk preferences and their robust representations. Math. Oper. Res. 38, 28-62 (2013)

14. Edgar, G.A., Sucheston, L.: Stopping Times and Directed Processes. Cambridge University Press, Cambridge (1992)

15. Ekeland, I., Témam, R.: Convex Analysis and Variational Problems. SIAM, Philadelphia (1999)

16. El Karoui, N., Ravanelli, C.: Cash subadditive risk measures and interest rate ambiguity. Math. Finance 19, 561-590 (2009) 
17. Farkas, W., Koch-Medina, P., Munari, C.: Capital requirements with defaultable securities. Insur. Math. Econ. (2013, forthcoming). arXiv:1203.4610 (May 2013)

18. Filipović, D., Kupper, M.: Monotone and cash-invariant convex functions and hulls. Insur. Math. Econ. 41, 1-16 (2007)

19. Föllmer, H., Schied, A.: Stochastic Finance: An Introduction in Discrete Time, 3rd edn. De Gruyter, Berlin (2011)

20. Frittelli, M., Rosazza Gianin, E.: Putting order in risk measures. J. Bank. Finance 26, 1473-1486 (2002)

21. Frittelli, M., Scandolo, G.: Risk measures and capital requirements for processes. Math. Finance 16, 589-612 (2006)

22. Hamel, A.H.: Translative sets and functions and their applications to risk measure theory and nonlinear separation. IMPA, Preprint Series D (2006). http://preprint.impa.br/FullText/Hamel_Fri_Mar_ 17_20_32_27_BRST_2006/acrm-main2.pdf

23. Hamel, A.H., Heyde, F., Rudloff, B.: Set-valued risk measures for conical market models. Math. Financ. Econ. 5, 1-28 (2011)

24. Jaschke, S., Küchler, U.: Coherent risk measures and good-deal bounds. Finance Stoch. 5, 181-200 (2001)

25. Kaina, M., Rüschendorf, L.: On convex risk measures on $L^{p}$-spaces. Math. Methods Oper. Res. 69, 475-495 (2009)

26. Konstantinides, D.G., Kountzakis, C.E.: Risk measures in ordered normed linear spaces with nonempty cone-interior. Insur. Math. Econ. 48, 111-122 (2011)

27. Krätschmer, V., Schied, A., Zähle, H.: Comparative and qualitative robustness for law-invariant risk measures. Finance Stoch. (2013, forthcoming). arXiv:1204.2458

28. Orihuela, J., Ruiz Galan, M.: Lebesgue property for convex risk measures on Orlicz spaces. Math. Financ. Econ. 6, 15-35 (2012)

29. Schaefer, H.H.: Banach Lattices and Positive Operators. Springer, Berlin (1974)

30. Shapiro, A., Dentcheva, D., Ruszczyński, A.: Lectures on Stochastic Programming: Modeling and Theory. SIAM, Philadelphia (2009)

31. Svindland, G.: Convex risk measures beyond bounded risks. PhD Dissertation, München (2008). http://edoc.ub.uni-muenchen.de/9715/ 